\title{
RADIO COUNTERPARTS OF COMPACT BINARY MERGERS DETECTABLE IN GRAVITATIONAL WAVES: A SIMULATION FOR AN OPTIMIZED SURVEY
}

\author{
K. НотоKezaka ${ }^{1}$, S. Nissanke ${ }^{2}$, G. Hallinan ${ }^{3}$, T. J. W. Lazio ${ }^{4}$, E. Nakar ${ }^{5}$, and T. Piran ${ }^{1}$ \\ ${ }^{1}$ Racah Institute of Physics, Hebrew University, Jerusalem, 91904, Israel \\ ${ }^{2}$ Institute of Mathematics, Astrophysics and Particle Physics, Radboud University, Heyendaalseweg 135, 6525 AJ Nijmegen, The Netherlands \\ ${ }^{3}$ Cahill Center for Astronomy, MC 249-17, California Institute of Technology, Pasadena, CA 91125, USA \\ ${ }^{4}$ Jet Propulsion Laboratory, California Institute of Technology, Pasadena, CA 91109, USA \\ ${ }^{5}$ Raymond and Beverly Sackler School of Physics \& Astronomy, Tel Aviv University, Tel Aviv 69978, Israel \\ Received 2016 May 30; revised 2016 August 25; accepted 2016 September 3; published 2016 November 8
}

\begin{abstract}
Mergers of binary neutron stars and black hole-neutron star binaries produce gravitational-wave (GW) emission and outflows with significant kinetic energies. These outflows result in radio emissions through synchrotron radiation. We explore the detectability of these synchrotron-generated radio signals by follow-up observations of $\mathrm{GW}$ merger events lacking a detection of electromagnetic counterparts in other wavelengths. We model radio light curves arising from (i) sub-relativistic merger ejecta and (ii) ultra-relativistic jets. The former produce radio remnants on timescales of a few years and the latter produce $\gamma$-ray bursts in the direction of the jet and orphan-radio afterglows extending over wider angles on timescales of weeks. Based on the derived light curves, we suggest an optimized survey at $1.4 \mathrm{GHz}$ with five epochs separated by a logarithmic time interval. We estimate the detectability of the radio counterparts of simulated GW-merger events to be detected by advanced LIGO and Virgo by current and future radio facilities. The detectable distances for these GW merger events could be as high as $1 \mathrm{Gpc}$. Around $20 \%-60 \%$ of the long-lasting radio remnants will be detectable in the case of the moderate kinetic energy of $3 \cdot 10^{50} \mathrm{erg}$ and a circum-merger density of $0.1 \mathrm{~cm}^{-3}$ or larger, while $5 \%-20 \%$ of the orphan-radio afterglows with kinetic energy of $10^{48} \mathrm{erg}$ will be detectable. The detection likelihood increases if one focuses on the well-localizable GW events. We discuss the background noise due to radio fluxes of host galaxies and false positives arising from extragalactic radio transients and variable active galactic nuclei, and we show that the quiet radio transient sky is of great advantage when searching for the radio counterparts.
\end{abstract}

Key words: gamma-ray burst: general - gravitational waves - radio continuum: stars - stars: neutron

\section{INTRODUCTION}

Joint electromagnetic (EM) and gravitational-wave (GW) observations of compact object mergers could allow for unprecedented measurements of astrophysical processes in strongly curved spacetimes. Such observations are possible thanks to a suite of advanced GW detectors and multiwavelength wide-field surveys that recently came online last year. A hundred years after Einstein's initial prediction of GWs, the first observations from binary black hole $(\mathrm{BH})$ mergers were measured simultaneously by the two LIGO detectors on 2015 September 14 (Abbott et al. 2016b) and 2016 December 26. These first detections mark a new era of transient-GW astronomy (see Abbott et al. 2016a for an overview and references therein). Aside from binary BHs, the other most numerous sources predicted for kilohertz $\mathrm{GW}$ detectors, such as LIGO (Abbott et al. 2009) and Virgo (Acernese et al. 2015) are the mergers of double neutron star (DNS) and black hole-neutron star (BH-NS) systems (The LIGO Scientific Collaboration et al. 2013). Through their dimensionless wavestrain $h(t)$, GWs encode key information about the progenitors' physical and geometric properties that probe the sources' bulk dynamic properties. These complicated mergers should also produce EM signatures from energetic matter outflows at different timescales. GW-EM detections will increase our confidence in detections of the GW signal and are essential for identifying the hosts and measuring their distances (Kochanek \& Piran 1993). Additionally, EM counterparts of mergers inform us about the environment, and the thermodynamic and composition properties of matter. We require both EM and GW measurements to fully understand neutron star binary mergers. In this paper, we focus on how to observe both GW and late radio emissions from these mergers.

Based on population synthesis models calibrated by observed Galactic DNS systems, advanced versions of GW detectors operating at their design sensitivity, such as LIGO, Virgo and KAGRA, expect to detect from 0.4 to 400 (with a mean value of 40) DNS mergers per year out to several hundred Mpc (Abadie et al. 2010; The LIGO Scientific Collaboration et al. 2013; Dominik et al. 2015). Such an expected rate is consistent with the observed short- $\gamma$ ray burst (sGRB) rate assuming a half-opening beaming angle of $\approx 10^{\circ}$ (Guetta \& Piran 2005, 2006; Nakar et al. 2006; Coward et al. 2012; Wanderman \& Piran 2015) and solar system abundance patterns of $r$-process elements (Bauswein et al. 2014; Piran et al. 2014; Hotokezaka et al. 2015; Shen et al. 2015; van de Voort et al. 2015; Wehmeyer et al. 2015; Vangioni et al. 2016). Based entirely on population synthesis models, since we have yet to observe a BH-NS system, we expect to detect 0.2-200 BH-NS systems per year with detectable distances of $\sim 1 \mathrm{Gpc}$.

For EM follow-up observations of compact object binary mergers, a fundamental challenge is the poor sky resolution of a single GW interferometer. Localizing a transient source on the sky depends primarily on triangulating the GW signal's arrival times using networks of three or more GW detectors (e.g., Fairhurst 2010). We must anticipate GW errors that span from 0.5 to $100 \mathrm{~s}$ square degrees sometimes with multimodal islands (e.g., Wen \& Chen 2010; Klimenko et al. 2011; Nissanke et al. 2011, 2013; Schutz 2011; Veitch et al. 2012; 
Grover et al. 2014; Rodriguez et al. 2014). The wide range depends on the sources' signal-to-noise ratio $(\mathrm{S} / \mathrm{N})$, their sky position, the number of detectors in the network and whether they are being used coherently. By exploiting the detectors' quadrupolar antenna functions, two or more GW detectors can also localize events from several hundred to a thousand square degrees within arcs of the sky (Kasliwal \& Nissanke 2014; Singer et al. 2014). Therefore EM observatories must be prepared for triggers from $\mathrm{GW}$ detectors that span these huge swaths of the sky of several hundred degrees released within minutes to thirty minutes of the merger being detected in GWs (e.g., Cannon et al. 2012; Singer et al. 2014).

At present several EM counterparts of DNS and BH-NS mergers have been proposed at different wavelengths and emission timescales. These include sGRBs and their afterglows (Eichler et al. 1989; Paczynski 1991; Narayan et al. 1992), optical-near-IR counterparts called macronovae or kilonovae (e.g., Li \& Paczyński 1998; Kulkarni 2005; Metzger et al. 2010; Roberts et al. 2011; Metzger \& Berger 2012; Barnes \& Kasen 2013; Berger et al. 2013; Tanaka \& Hotokezaka 2013; Tanvir et al. 2013; Yang et al. 2015; Jin et al. 2016) and longlasting radio merger remnants (Nakar \& Piran 2011; Piran et al. 2013; Hotokezaka \& Piran 2015). Additional radio emission and, in particular, a coherent prompt radio pulse from a magnetically driven, relativistic plasma outflow prior to the DNS merger have also been proposed (Hansen \& Lyutikov 2001; Pshirkov \& Postnov 2010; Palenzuela et al. 2013; Totani 2013). These prompt radio counterparts are both harder to detect and less certain than the above three candidates for DNS mergers, so we do not discuss these in this paper; see Chu et al. (2015) for a detailed study.

The radio counterparts of NS merger events promise to uniquely probe their energetics and environment. The interaction of the merger ejecta with the circum-merger medium results in a radio remnant with timescales of a few months to years (Nakar \& Piran 2011). The radio luminosity can be brighter by a couple of orders of magnitude than that of a typical radio supernova. Although such radio remnants have not yet been observed after sGRBs (e.g., Metzger \& Bower 2014; Horesh et al. 2016), they may be detectable by follow-up observations for GW merger events since they will take place at much closer distances than sGRBs. In addition, the sGRB jet produces a radio afterglow with a timescale of a few weeks, which can be seen provided that the viewing angle is not too large. In fact, radio afterglows have been already detected for four sGRBs (e.g., Fong et al. 2015) and they will be detectable counterparts of GW merger events (Feng et al. 2014). Recent observations suggest that the radio transient sky is far quieter compared to the optical one, so we expect far fewer false positives by a factor of a hundred or more depending on the sky location than in the optical-IR (e.g., Frail et al. 2012; Mooley et al. 2013, 2016).

This paper focuses on strategies for detecting and identifying, following the detection of a GW event, two different radio post-merger counterparts: (i) the longer duration radio remnants that may last from months to years and (ii) the faster ultrarelativistic radio afterglow components that last weeks to months (Nakar \& Piran 2011; Piran et al. 2013; Hotokezaka \& Piran 2015; Margalit \& Piran 2015). Importantly, we do not consider here the ideal case in which a sGRB or an optical counterpart to a GW merger has been detected. Note that a sGRB and its bright afterglow are expected only for on-axis events, which are most likely a small fraction of all events. In this case, the high-energy signal will immediately provide a much better localization, enabling very accurate radio identification of the merger's position.

To define an optimal strategy for detecting radio counterparts, we run a simulation that considers both $\mathrm{GW}$ and radio detectability of neutron star mergers with a slew of GW and radio telescopes. Our simulation comprises six steps and the structure of the paper follows these.

(i) We construct underlying astrophysically motivated distributions of DNS and BH-NS (with a $5 M_{\odot} \mathrm{BH}$ ) mergers (Section 2.1).

(ii) We then simulate GW wavestrains $h(t)$ for each binary in our underlying catalog and ask whether the wavestrain is detectable with different GW networks (Sections 2.2 and 2.3).

(iii) For a random subset of GW detectable sources we estimate the entire set of source parameters including the sky position, distance and inclination angle to each binary (Sections 2.2 and 2.3).

(iv) We perform an approximate mapping from the binary progenitor to the dynamical mass ejecta and ultrarelativistic outflows using a range of numerical-relativity and smooth particle hydrodynamic (SPH) simulations (Section 3.2).

(v) We compute different radio afterglow and remnant signatures for each $\mathrm{GW}$ detectable binary using our set of ejecta models (Section 3.3).

(vi) We check whether the different radio signatures are detectable by a slew of radio telescopes at different frequencies (Section 4).

Following these steps we outline the challenges to ensure a secure identification of a GW-radio counterpart among other astrophysical transients and suggest an optimal strategy to overcome them (Section 5). We summarize the results, compare them with previous work (Section 6) and conclude with a discussion of their implications (Section 8).

\section{GW DETECTABILITY OF MERGERS}

We begin by describing how we construct NS binary merger catalogs and the methods used to derive sky location, distance and inclination angle measures for populations of DNS or BHNS binary mergers detectable in GWs. We first outline the schema of our method. Based on Sections 2-5 of Nissanke et al. (2013) (henceforth denoted NKG13), we then describe the technical aspects of simulating the anticipated sky positions and distance measurements.

\subsection{Catalogs of NS Binary Populations}

We construct two underlying astrophysical NS binary catalogs with either $4 \times 10^{4} \mathrm{DNS}$ or $3 \times 10^{4} \mathrm{NS}-5 M_{\odot} \mathrm{BH}$ populations. In this section, we do not take into account DNS and BH-NS merger rates or GW detection volumes (see Section 2.2). However, each binary in our two catalogs is described by the nine parameters that are encoded in the theoretical predictions of the GW wavestrain (or GW waveform) for non-spinning compact binary systems (Blanchet 2014 and references therein). As discussed in Section 2.3, these parameters are critical to the GW detectability as the GW waveform depends on them. They include the two compact 
object masses $m_{1}$ and $m_{2}$, a sky position $\boldsymbol{n} \equiv(\theta, \phi)$, a luminosity distance $D_{L}$, an inclination angle $\iota$, a polarization angle $\psi$ and a time and phase of the GW merger. The colatitude $\theta$ and longitude $\phi$ are related to the declination $\delta$ and right ascension $\alpha$, by $\theta=\pi / 2-\delta$ and $\phi=\alpha-$ GAST respectively, where GAST is Greenwich Apparent Sidereal Time. Apart from the component masses, the luminosity distance and the time of merger, we assume random distributions in the other parameters: $p(\cos \iota) \propto$ const with $\cos \iota \in[-1,1]$, and a random sky position such that $p(\cos \theta) \propto$ const with $\cos \theta \in[-1,1]$, and $p(\phi) \propto$ const with $\phi \in[0,2 \pi]$. We set the time of merger to be a constant value.

We specify NS and BH component masses of $1.4 M_{\odot}$ and $5 M_{\odot}$ respectively. As indicated by SPH and numericalrelativity simulations, we choose these particular values in masses such that the mergers of the two objects will form some dynamic ejecta and the NS will not be swallowed entirely by the gravitational potential of a too massive $\mathrm{BH}$ (see e.g., Foucart 2012; Kawaguchi et al. 2015; Kyutoku et al. 2015). The ejecta create matter outflows and therefore are potentially responsible for radio afterglows and long-lasting radio remnants (Nakar \& Piran 2011; Piran et al. 2013; Hotokezaka $\&$ Piran 2015). For simplicity, we assume in our simulations that the NSs and BHs are non-spinning; in the physical universe we expect $\mathrm{BHs}$ in $\mathrm{BH}-\mathrm{NS}$ systems to have a considerable spin, so there may be more dynamical ejecta produced for a NS orbiting in a prograde orbit around a $\mathrm{BH}$ with a maximal spin. Furthermore, modulo selection effects and the small sample number, observations of Galactic DNS systems imply a narrow mass distribution for them. We assume that the DNS and BH-NS systems chosen in our catalogs will merge within the Hubble time.

For systems with distances $<200 \mathrm{Mpc}$, we assume that the spatial distribution of neutron star binaries traces host galaxy light. As described in NKG13, we correct for B-band luminosity using the "Census of the Local universe" (CLU) with information compiled from different galaxy catalogs (e.g., HyperLEDA, NED, EDD; see Kasliwal 2011 and Gehrels et al. 2016 for details). For those systems located with distances $>200 \mathrm{Mpc}$, we assume that the NS binary merger distribution follows a constant comoving volume density in a $\Lambda$ CDM universe (Komatsu et al. 2009).

\subsection{Gravitational-wave Detector Networks}

We consider different GW networks comprising the advanced versions of LIGO, Virgo, KAGRA and LIGO India; see The LIGO Scientific Collaboration et al. (2013) for a observing scenario roadmap of the LIGO and Virgo detectors over the next decade. Each detector will operate in staggered science modes with increasing sensitivity until they reach their target design sensitivity. For instance, advanced LIGO began its first observation run in 2015 September, advanced Virgo could begin its first observation run as early as 2016 September, and KAGRA could begin as early as 2019. We make several assumptions in this work; for instance, we assume Gaussian, stationary, and zero-mean noise that is independent and uncorrelated between detectors. We also take the anticipated noise sensitivity curve for a single advanced LIGO detector, given in The LIGO Scientific Collaboration et al. (2013), for broadband tuning, to be representative of all our detectors, imposing a low-frequency cut off of $10 \mathrm{~Hz}$; see Berry et al. (2015) for parameter inference methods using non-
Gaussian, non-stationary noise. In reality, LIGO, Virgo, and KAGRA will have different noise sensitivities in different frequency bands because of variations in each instrument's design, and systematic calibration effects must be taken into effect.

In the rest of the paper, we use the following notation to describe different GW networks with $N$ detectors: $G W$ Net 3 or GW network 3 comprises LIGO Hanford, LIGO Livingston and Virgo, and Net5 or network 5 consists of LIGO Hanford, LIGO Livingston, Virgo, LIGO India and KAGRA. See the recent works of Singer et al. (2014), Berry et al. (2015), Kasliwal \& Nissanke (2014) for sky location works using either only two LIGO detectors or the three LIGO-Virgo detectors at staggered (and not full) design sensitivities.

To implement a detectability criterion, we assume that each merger in the two catalogs is detectable in GWs if its GW expected network $\mathrm{S} / \mathrm{N}>8.5$ (see Section 3.3 in NKG13 for different triggering criteria and GW networks).

\subsection{Extracting the Binaries' Sky Location, Luminosity Distance and Inclination Angle from the GW Signal}

Based on optimal matched filtering (Finn 1992; Cutler \& Flanagan 1994), we extract the luminosity distance $D_{L}$, the inclination angle $\cos \iota$, and sky position $\boldsymbol{n}$ for each DNS or $\mathrm{BH}-\mathrm{NS}$ binary merger using knowledge of the expected $\mathrm{GW}$ signal.

Regarding the predicted GW waveform, we use only the early inspiral (pre-merger) portion of the waveform, which for low-mass systems provides most of the signal for advanced detectors (Flanagan \& Hughes 1998). The inspiral waveform is modeled accurately using post-Newtonian (PN) expansions in general relativity and is based on an expansion in $\sim v^{2} / c^{2}$, where $v$ is the characteristic orbital speed for gravitationally bound systems (Blanchet 2014). Specifically, we use the nonspinning restricted $3.5 \mathrm{PN}$ waveform in the frequency domain for the two GW polarizations $h_{+}$and $h_{\times}$; see Equation (1) in NKG13 and Equations (7)-(14) in Nissanke et al. (2010). The overall Newtonian amplitude of the GW waveform encodes the source's orientation, sky location, luminosity distance and redshifted chirp mass. The GW phase depends on the redshifted chirp mass, redshifted reduced mass, the phase and time of merger. The detector antenna functions depend on $\boldsymbol{n}$ and the binary's polarization angle. The time of flight from source at direction $\boldsymbol{n}$ to detector at location $\boldsymbol{r}$ involves the scalar product $\boldsymbol{n} \cdot \boldsymbol{r}$, and differences in time of flight between detectors in the network dominate how well we can localize the event on the sky.

To infer the sky position $(\cos \theta, \phi)$, luminosity distance $D_{L}$, and $\cos \iota$, we explicitly map out the posterior probability density function for all source parameters (including chirp mass, orientation, etc.) after simulating a data stream at a detector using Markov Chain Monte Carlo (MCMC) methods. The Metropolis-Hastings MCMC algorithm used is based on a generic version of CosmoMC, described in Lewis \& Bridle (2002), and is detailed in Section 3.3 of Nissanke et al. (2010). Other parameter estimation methods used frequently in the LIGO-Virgo analysis pipelines are summarized in Veitch et al. (2015) and The LIGO Scientific Collaboration \& the Virgo Collaboration (2016).

We take prior distributions in all source parameters to be flat over the region of sample space where the binary is detectable at an expected network $\mathrm{S} / \mathrm{N}=3.5$. This $\mathrm{S} / \mathrm{N}$ is defined as the 
root-sum-square of the expected individual detector S/Ns. For each MCMC simulation, we derive distance and inclination angle measures, and solid angle areas over $(\cos \theta, \phi)$ for $68 \%$, $95 \%$, and $99 \%$ confidence regions.

\section{RADIO SIGNATURES OF COMPACT BINARY MERGERS}

Radio emission from post-merger events are produced via synchrotron radiation of accelerated electrons in shocks formed between expanding outflows and circum-merger material (Nakar \& Piran 2011; Piran et al. 2013; Hotokezaka \& Piran 2015). We briefly review synchrotron radiation from sub-relativistic and ultra-relativistic outflows. We then provide our models of radio emission arising from these outflows.

\subsection{Synchrotron Radiation of Expanding Outflows}

We turn now to estimate the properties of radio signals arising from outflows expanding into homogeneous circummerger material. We consider first long-lasting radio remnants arising from sub-relativistic merger ejecta and then orphan GRB afterglows arising from ultra-relativistic jets.

Long-lasting radio remnants. The outflow expands with an initial velocity until the kinetic energy of the swept-up material is comparable to the ejecta's own kinetic energy. For ejecta thrown out at mildly and sub-relativistic speeds, the deceleration timescale is given by (e.g., Nakar \& Piran 2011)

$$
t_{\mathrm{dec}} \approx 80 \text { day } E_{50}^{\frac{1}{3}} n^{-\frac{1}{3}} \beta_{0}^{-\frac{5}{3}},
$$

where $E$ is the kinetic energy of the ejecta, $\beta_{0}$ is the ejecta's initial velocity in units of the speed of light, and $n$ is the circum-merger density. Here and elsewhere the notation $Q_{x}$ indicates the value of the variable $Q / 10^{x}$ in cgs units. The peak times of the light curves arising from the ejecta are scaled with Equation (1).

Electrons are accelerated in shocks between the ejected outflow and the circum-merger material and emit synchrotron radiation. Here we assume a power-law electron distribution with an index $p$. The characteristic frequency of the synchrotron radiation is:

$$
\nu_{m} \approx 1 \mathrm{GHz} n^{1 / 2} \epsilon_{B,-1}^{1 / 2} \epsilon_{e,-1}^{2} \beta^{5},
$$

where $\beta$ is the ejecta's velocity, $\epsilon_{e}$ and $\epsilon_{B}$ are the conversion efficiencies from the shock's internal energy into the energy of the accelerated electrons and magnetic field respectively. The synchrotron spectra have a maxima at $\nu_{m}$ as long as $\nu_{m}$ is above the synchrotron self-absorption frequency (see below). After $t_{\mathrm{dec}}, \nu_{m}$ decreases with time and the flux density at a given frequency above $\nu_{m}$ declines with time. The peak flux density above $\nu_{m}$ is estimated as:

$$
\begin{aligned}
F_{\text {peak }, \nu>\nu_{m}} \approx & 8 \text { mJy } E_{50} n^{\frac{p+1}{4}} \epsilon_{B,-1}^{\frac{p+1}{4}} \epsilon_{e,-1}^{p-1} \beta_{0}^{\frac{5 p-7}{2}} \\
& \times\left(\frac{D_{L}}{200 \mathrm{Mpc}}\right)^{-2}\left(\frac{\nu}{1.4 \mathrm{GHz}}\right)^{-\frac{p-1}{2}},
\end{aligned}
$$

\footnotetext{
6 One may find that the value of $t_{\mathrm{dec}}$ estimated here is slightly different from that in Nakar \& Piran (2011). This is because we round off the number while Nakar \& Piran (2011) round the value down.
}

where $\nu$ is the observation frequency, and $D_{L}$ is the luminosity distance to the source. In this work we set the values of the parameters: $\epsilon_{e}=\epsilon_{B}=0.1$ and $p=2.5$. This choice is motivated by observations of late radio afterglows in long GRBs and typical radio supernovae (Chevalier 1998; Frail et al. 2000, 2005).

The above estimates of the peak timescale and flux density are valid only when synchrotron self-absorption is unimportant. In the case of either sufficiently high circum-merger densities or low observation frequencies, such absorption can be important. The synchrotron self-absorption frequency $\nu_{a}$ at $t_{\mathrm{dec}}$ is estimated as:

$$
\begin{aligned}
\nu_{a, \mathrm{dec}} \approx & 1.3 \mathrm{GHz} E_{50}^{\frac{2}{3(p+4)}} n^{\frac{3 p+14}{6(p+4)}} \epsilon_{B,-1}^{\frac{2+p}{2(p+4)}} \\
& \times \epsilon_{e,-1}^{\frac{2(p-1)}{p+4}} \beta_{0}^{\frac{15 p-10}{3(p+4)}} .
\end{aligned}
$$

Below $\nu_{a \text {,dec }}$, the peak flux density and peak timescale are estimated as:

$$
\begin{aligned}
F_{\text {peak }, \nu<\nu_{a}} \approx & 0.6 \text { mJy } E_{50}^{\frac{4}{5}} n^{\frac{1}{5}} \epsilon_{B,-1}^{\frac{1}{5}} \epsilon_{e,-1}^{\frac{3}{5}} \\
& \times\left(\frac{D_{L}}{200 \mathrm{Mpc}}\right)^{-2}\left(\frac{\nu}{150 \mathrm{MHz}}\right)^{\frac{6}{5}},
\end{aligned}
$$

and

$$
\begin{aligned}
t_{\nu<\nu_{a}\left(t_{\mathrm{dec}}\right)} \approx & 570 \text { day } E_{50}^{\frac{5}{11}} n^{\frac{7}{22}} \epsilon_{B,-1}^{\frac{9}{22}} \epsilon_{e,-1}^{\frac{6}{11}} \\
& \times\left(\frac{\nu}{150 \mathrm{MHz}}\right)^{-\frac{13}{11}} .
\end{aligned}
$$

Orphan GRB afterglows. Compact binary mergers produce ultra-relativistic jets that result in sGRBs (Eichler et al. 1989; Nakar 2007). Jets produce not only prompt gamma-ray emission but also afterglows at longer wavelength as a result of the interaction with the circum-merger material (e.g., Sari et al. 1998). For relativistic jets, the emission is highly beamed toward the jet axis, which has an important consequence for detectability. Observers only on or close to the jet axis can measure its bright emission. In contrast, observers far away from the jet axis can measure the faint emission only after the jet's sufficient deceleration and its subsequent interaction with its environment. Therefore the observed light curves depend strongly on the observers' viewing angle. Roughly speaking, the peak timescale of the relativistic radio afterglow for an offaxis observer occurs when $\Gamma \sim \theta_{\text {obs }}^{-1}$, where $\Gamma$ is the jet's Lorentz factor and $\theta_{\text {obs }}$ is the observer's viewing angle.

It is worth noting that the spectral shapes of GRB afterglows are different from those of the long-lasting radio remnants. For GRB afterglows, as the characteristic frequency decreases with time because of the relation $\nu_{m} \propto \Gamma^{4}$ during the jet's deceleration, observers detect synchrotron radiation from higher (e.g., X-ray) to lower multi-wavelength frequencies. When $\nu_{m}$ decreases to radio frequencies, the Lorentz factor, i.e., the beaming factor, is sufficiently low that off-axis observers can detect the late-time radio signals from the jet. For observers on or close to the jet axis, observing at higher radio frequencies (e.g., $5 \mathrm{GHz}$ ) is preferable for avoiding flux losses due to synchrotron self-absorption. In contrast, for long-lasting radio remnants and off-axis orphan afterglows, observing at lower radio frequencies is preferable because the characteristic frequency $\nu_{m}$ is typically lower than $1 \mathrm{GHz}$ for sub-relativistic 
Table 1

The Energetic Properties of DNS and BH-NS Merger Ejecta from Different Sets of Numerical Simulations (the Notation GR Refers to a Fully General Relativistic Simulation, CF to a Conformally Flat Simulation, Newton to a nonRelativistic Simulation, Mesh to a Grid-based Hydrodynamics and SPH to Smooth Particle Hydrodynamics)

\begin{tabular}{lcclr}
\hline \hline Type & Range of $\beta_{\text {ave }}$ & Range of $E\left(10^{50} \mathrm{erg}\right)$ & Scheme & Ref. \\
\hline DNS & $0.15,0.3$ & $0.1,10$ & GR, Mesh & $(1)$ \\
DNS & $0.15,0.4$ & $0.5,10$ & CF, SPH & $(2)$ \\
DNS & $0.1,0.15$ & 2,10 & Newton, SPH & $(3)$ \\
BH-NS & $0.2,0.3$ & $10^{-3}, 60$ & GR, Mesh & $(4)$ \\
BH-NS & $0.2,0.25$ & 10,40 & CF, SPH & $(5)$ \\
BH-NS & $0.15,0.2$ & 6,20 & Newton, SPH & $(3)$
\end{tabular}

References. (1) Hotokezaka et al. (2013), Sekiguchi et al. (2015), Radice et al. (2016), (2) Bauswein et al. (2013), (3) Rosswog (2013), Piran et al. (2013), (4) Foucart et al. (2013), Kyutoku et al. (2015), Kawaguchi et al. (2015), (5) Just et al. (2015).

ejecta and off-axis afterglows. Therefore, we focus mainly on frequencies of $150 \mathrm{MHz}$ and $1.4 \mathrm{GHz}$ in the rest of this work.

\subsection{An Approximate Mapping from Progenitor to Outflow Models}

Various components of merger outflows may be produced with different velocities and kinetic energies. We consider here the expected range in energetics for the two post-merger radio counterparts that give rise to the most luminous radio remnants and are arguably the most robust (see Hotokezaka \& Piran 2015) : (i) the long-lasting isotropic radio remnant that varies from a year to ten year timescale, and (ii) the afterglow of an ultra-relativistic GRB jet that varies on a week to month timescale. Below we detail how we model these two outflows using the results of numerical-relativity and SPH simulations of the NS mergers themselves.

\section{Dynamical Mass Ejection}

DNS mergers. We expect mergers to produce tidal tails which result in gravitationally unbound dynamical mass ejecta that undergo shock heating processes. For a binary with given component masses and different NS equation of state, we estimate the range of ejecta kinetic energy and velocity using results that span a diverse set of numerical relativity and SPH simulations (Bauswein et al. 2013; Hotokezaka et al. 2013; Rosswog 2013; Sekiguchi et al. 2015, 2016; Radice et al. 2016). Table 1 lists the range of the kinetic energy and average velocity for DNS mergers from the literature. Within these uncertainties, the majority of the simulations exhibit ejecta kinetic energies in the range of $10^{50} \lesssim E \lesssim 10^{51} \mathrm{erg}$ and average velocities of $0.2 \lesssim \beta \lesssim 0.3$. To incorporate model uncertainties in $E$ and $\beta$, we henceforth define three models, named $\mathrm{DNS}_{\mathrm{h}}, \mathrm{DNS}_{\mathrm{m}}$, and $\mathrm{DNS}_{\mathrm{l}}$, where the indices $\mathrm{h}, \mathrm{m}$, and $\mathrm{l}$ stand for typical high, median and low values of the energy and velocity parameter space that our representative set of numerical simulations span (see Table 1). Table 2 shows the kinetic energy and average velocity for each model.

$B H-N S$ mergers. In a BH-NS merger we expect dynamical ejecta through the tidal disruption of the NS by the BH's gravitational potential. The amount of ejecta depends on the asymmetry of the BH-NS system, namely the individual masses, the BH's spin and the NS equation of state (e.g., Rosswog 2005, 2013; Foucart et al. 2013; Piran et al. 2013; Just et al. 2015; Kawaguchi et al. 2015; Kyutoku et al. 2015). A
Table 2

The Mean Energetics Chosen for our Different Ejecta Models in the Case of DNS, BH-NS, and sGRB-jet Driven Events

\begin{tabular}{lccccc}
\hline \hline Model & $E_{K}(\mathrm{erg})$ & $\left\langle\beta_{0}\right\rangle(c)$ & $\begin{array}{c}L_{1.4 \mathrm{GHz}}^{n=1} \\
\left(\mathrm{erg} \mathrm{s}^{-1} \mathrm{~Hz}^{-1}\right)\end{array}$ & $L_{1.4 \mathrm{GHz}}^{n=0.1}$ & $L_{1.4 \mathrm{GHz}}^{n=0.01}$ \\
\hline DNS $_{h}$ & $10^{51}$ & 0.3 & $4 \cdot 10^{29}$ & $8 \cdot 10^{28}$ & $10^{28}$ \\
DNS $_{m}$ & $3 \cdot 10^{50}$ & 0.25 & $8 \cdot 10^{28}$ & $10^{28}$ & $2 \cdot 10^{27}$ \\
DNS $_{l}$ & $10^{50}$ & 0.2 & $10^{28}$ & $2 \cdot 10^{27}$ & $3 \cdot 10^{26}$ \\
BH-NS $_{h}$ & $5 \cdot 10^{51}$ & 0.3 & $2 \cdot 10^{30}$ & $5 \cdot 10^{29}$ & $7 \cdot 10^{28}$ \\
BH-NS $_{m}$ & $2 \cdot 10^{51}$ & 0.25 & $5 \cdot 10^{29}$ & $8 \cdot 10^{28}$ & $10^{28}$ \\
BH-NS $_{l}$ & $5 \cdot 10^{50}$ & 0.2 & $7 \cdot 10^{28}$ & $9 \cdot 10^{27}$ & $10^{27}$ \\
strong-jet & $10^{49}$ & $\sim 1$ & $3 \cdot 10^{28}$ & $10^{28}$ & $2 \cdot 10^{27}$ \\
canonical-jet & $10^{48}$ & $\sim 1$ & $4 \cdot 10^{27}$ & $10^{27}$ & $2 \cdot 10^{26}$ \\
\hline
\end{tabular}

Note. For the jet models, we assume a viewing angle of $45^{\circ}$. The 4th-6th columns show the radio peak luminosities at $1.4 \mathrm{GHz}$ with the circum-merger densities of $1,0.1$, and $0.01 \mathrm{~cm}^{-3}$.

high spin parameter and a large NS radius should result in a larger amount of ejecta. It is worth emphasizing that BH-NS mergers could eject larger amounts of mass than DNS mergers. For instance, recent numerical relativity simulations show that for extreme tidal disruption cases, masses of $\sim 0.05 M_{\odot}$ can be ejected with velocities in the range of $\beta \sim 0.2-0.3$ (Foucart et al. 2013; Kyutoku et al. 2015). Here we focus only on the cases where the tidal disruption is sufficiently strong and define three different models to encompass the range in energetics: $\mathrm{BH}-\mathrm{NS}_{\mathrm{h}}, \mathrm{BH}-\mathrm{NS} \mathrm{m}_{\mathrm{m}}$, and $\mathrm{BH}-\mathrm{NS}_{1}$ (see the kinetic energy and average velocity of each model in Table 2). Note that although we consider only NS-5 $M_{\odot}$ BH mergers for simulating GW detections, the kinetic energies and velocities used here somewhat cover the ejecta of massive BH-NS mergers, e.g., a BH mass of $10 M_{\odot}$.

Numerical simulations show that the ejecta velocity is not a single value but follows a power law toward the high-velocity limit $\beta \gtrsim 0.5$. Here we assume a velocity distribution function which can broadly describe the results of numerical simulations as (see the Appendix):

$$
\frac{d M}{d \beta}=\frac{M_{0}\left(\frac{\beta}{\beta_{a}}\right)^{-\alpha}}{1+\exp \left(\left(\beta-\beta_{c}\right) / \sigma_{c}\right)},
$$

where we choose the parameters $\alpha=-1$ for $\beta<\beta_{a}, \alpha=2.5$ for $\beta \geqslant \beta_{a}, \beta_{c}=2 \beta_{a}$, and $\sigma_{c}=0.035$, as motivated by Hotokezaka et al. (2013). $M_{0}$ and $\beta_{a}$ are the parameters which control the values of the kinetic energy and average velocity. The exact form of the high-velocity cutoff is unclear because it is difficult to resolve the dynamics of such a small amount of ejecta. Recently, from the results of a GR-SPH simulation by Bauswein et al. (2013), Metzger et al. (2015a) found free neutron components of the ejecta mass extending to high velocities $\beta \gtrsim 0.8$ in DNS ejecta (see also Kyutoku et al. 2014 for an analytic argument). If this component physically exists, radio luminosities of DNS ejecta should be brighter than our results given here at earlier times (Hotokezaka \& Piran 2015).

As the ejecta's velocity distribution is non-uniform, we estimate the emission from each shell of matter and combine the results (Piran et al. 2013). For a given kinetic energy distribution in velocity space, we divide the outflow into spherical shells. The circum-merger material with a mass of $M(R)$ swept up at a radius $R$ can be associated with each shell 
such that this mass slows down the shells:

$$
M(R)(c \beta \Gamma)^{2}=E(\geqslant \beta \Gamma) .
$$

Once we implicitly solve Equation (8), we are able to determine the observable light curves. We then combine the contributions of the different shells to obtain the total light curve. Note that, in this work, we do not take non-spherical geometry of the ejecta into account. The asphericity does not affect the radio fluxes significantly but it will delay the peak timescales (Margalit \& Piran 2015).

Ultra-relativistic jet. An ultra-relativistic jet travels with the initial Lorentz factor and the initial jet half-opening angle in the external medium until the total energy of the swept-up material becomes comparable to the jet's initial kinetic energy. After this stage, the jet slows down but remains relativistic and maintains the initial jet's opening angle. The radiation from the jet is thus still collimated. Once the jet's Lorentz factor becomes roughly $\theta_{j}^{-1}$, the jet starts the sideway expansion and approaches to a fully spherical expansion. During this stage, the jet's radiation begins to be decollimated and detectable to off-axis observers (see Granot et al. 2002; Nakar et al. 2002; van Eerten et al. 2010; De Colle et al. 2012 for details of offaxis afterglow light curves).

We follow the jet dynamics using a semi-analytic model proposed by Granot \& Piran (2012), which can approximately reproduce the jet evolution resulting from a numerical simulation by De Colle et al. (2012). Once we compute the jet dynamics, we derive the afterglow synchrotron radiation at each observer's time (Sari et al. 1998; Granot et al. 1999). We choose the jet parameters, the initial half-opening angle and the kinetic energy based on the observations of sGRBs. The initial jet's half-opening angle is measured from the chromatic break in the afterglow light curves. While there are significant uncertainties in estimates of $\theta_{j}$ from observations, we set the initial jet half-opening angle to be $10^{\circ}$ (Fong et al. 2014).

As with the long-lasting radio remnant, we choose two different jet models: the canonical-jet model has a kinetic energy of $10^{48} \mathrm{erg}$ and the strong-jet model has a corresponding value of $10^{49} \mathrm{erg}$ (see Table 2 ). We choose the values for the kinetic energies because the isotropic equivalent $\gamma$-ray energy of sGRBs is in the range from $10^{49}$ to $10^{51} \mathrm{erg}$ (Nakar 2007). Assuming that the kinetic energy of the jet is comparable to the $\gamma$-ray energy and taking into account a jet beaming angle of $10^{\circ}$, the jets' kinetic energies are $10^{47} \sim 10^{50} \mathrm{erg}$. We choose $10^{48} \mathrm{erg}$ as a canonical value since there are more events in the lower energy range according to the luminosity function of sGRBs (Wanderman \& Piran 2015).

\subsection{Radio Light Curves}

In this section, we explicitly show the expected light curves for our radio counterpart models assuming different circummerger densities $n=1.0, \quad 0.1 \mathrm{~cm}^{-3}, \quad 0.01 \mathrm{~cm}^{-3}$ and $0.001 \mathrm{~cm}^{-3}$.

Figure 1 shows the radio light curves of the DNS models (left panels) and BH-NS models (right panels) at $1.4 \mathrm{GHz}$ (upper panels) and $150 \mathrm{MHz}$ (lower panels). Also shown are the light curves of strong-jet and canonical-jet sGRB models with three different viewing angles of $30^{\circ}, 45^{\circ}$, and $60^{\circ}$. We set the luminosity distances to be $200 \mathrm{Mpc}$ and $300 \mathrm{Mpc}$ for DNS and BH-NS respectively (e.g., NKG13 and
The LIGO Scientific Collaboration et al. 2013). In addition, as we discuss in Section 4, we show the $7 \sigma$ root-mean-square (rms) noise level of the radio facilities considered with integration of one hour and the flux densities at $1.4 \mathrm{GHz}$ of fiducial galaxies: the Milky Way and M82, assuming an observer at a distance of $200 \mathrm{Mpc}$ for DNS mergers and $300 \mathrm{Mpc}$ for $\mathrm{BH}-\mathrm{NS}$ mergers. Here we show the peak flux density of the edge-on Milky Way for ASKAP (see Section 5.1).

The radio peak flux density of each model is in the range of $\sim 0.01 \mathrm{mJy}$ to a few mJy. However, the long-lasting radio remnants and orphan afterglows have different timescales. The orphan afterglows peak at early times, between a week and a month, depending on the viewing angle, on the jets' kinetic energy, and on the circum-merger density. The long-lasting radio remnants peak at late times (a few hundred days). Roughly speaking, for generic observers $\theta_{v} \sim 45^{\circ}$, the strongjet and canonical-jet afterglows are as bright as $\mathrm{DNS}_{\mathrm{m}}$ and $\mathrm{DNS}_{1}$ at $1.4 \mathrm{GHz}$ respectively. At $150 \mathrm{GHz}$, the peak flux densities from the long-lasting radio remnants are higher than at $1.4 \mathrm{GHz}$ and their timescales are longer. In contrast, the orphan afterglows at $150 \mathrm{MHz}$ are significantly fainter because of the synchrotron self-absorption.

Figure 2 shows the dependence of the radio flux density on the circum-merger density. Here we show only $\mathrm{DNS}_{\mathrm{m}}, \mathrm{BH}-$ $\mathrm{NS}_{\mathrm{m}}$, and canonical jet with a viewing angle of $45^{\circ}$. At $1.4 \mathrm{GHz}$ (above the self-absorption frequency in these cases), the flux densities are sensitive to the circum-merger density as $F \propto n^{(p+1) / 4}$. On the other hand, at $150 \mathrm{MHz}$, these depend only weakly on the density as long as the self-absorption frequency is higher than $150 \mathrm{MHz}$. At densities below $0.1 \mathrm{~cm}^{-3}$, the peak fluxes of the long-lasting radio remnants significantly decrease with densities since synchrotron selfabsorption is less important.

The peak times and flux densities of the long-lasting radio remnants are faster and larger by a factor of $\sim 2$ than those in Piran et al. (2013). One of the reasons is that we take the faster ejecta velocities based on numerical relativity simulations (see Equation (3) for a strong dependence of the peak flux density on the velocity), while Piran et al. (2013) use a Newtonian SPH simulation. Furthermore, when calculating the light curves, we incorporate relativistic effects, the Doppler effect and relativistic beaming, which also result in slightly faster and brighter light curves even for mildly relativistic velocities.

In order to optimize the detectability of orphan radio afterglows and the long-lasting radio remnants of GW mergers, based on our predicted light-curves (see Figures 1 and 2), we provide suggested epochs of follow-up observations that are roughly separated by logarithmic time intervals:

(1) Reference imaging of the GW error area or GW area occupied by galaxies within a day after the GW detection.

(2) Observing the peak timescale of the canonical orphan afterglows and the rise in the light curve of bright orphan afterglows at $\lesssim 10$ days.

(3) Observing the decline in canonical orphan afterglows and the peak in strong afterglow signatures at $\sim 30$ days.

(4) Observing the fading of strong orphan afterglows and the rise in the light curves of long-lasting radio remnants at $\sim 100$ days.

(5) Observing the peak in the long-lasting radio remnants at $\sim 300$ days. 
DNS, $1.4 \mathrm{GHz}, \mathrm{D}=200 \mathrm{Mpc}, \mathrm{n}=0.1 \mathrm{~cm}^{-3}$

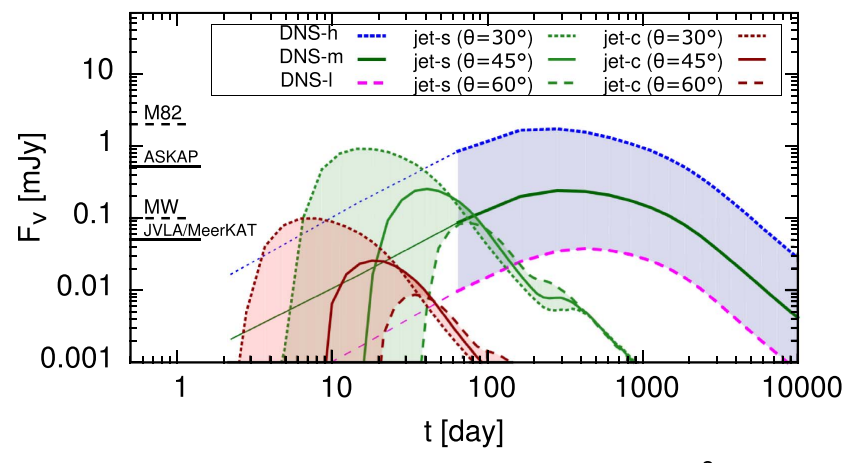

DNS, $150 \mathrm{MHz}, \mathrm{D}=200 \mathrm{Mpc}, \mathrm{n}=0.1 \mathrm{~cm}^{-3}$

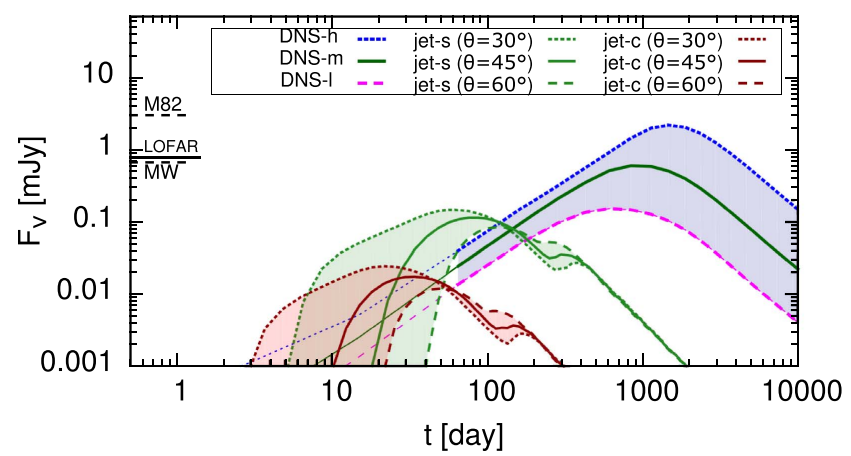

BH-NS, $1.4 \mathrm{GHz}, \mathrm{D}=300 \mathrm{Mpc}, \mathrm{n}=0.1 \mathrm{~cm}^{-3}$

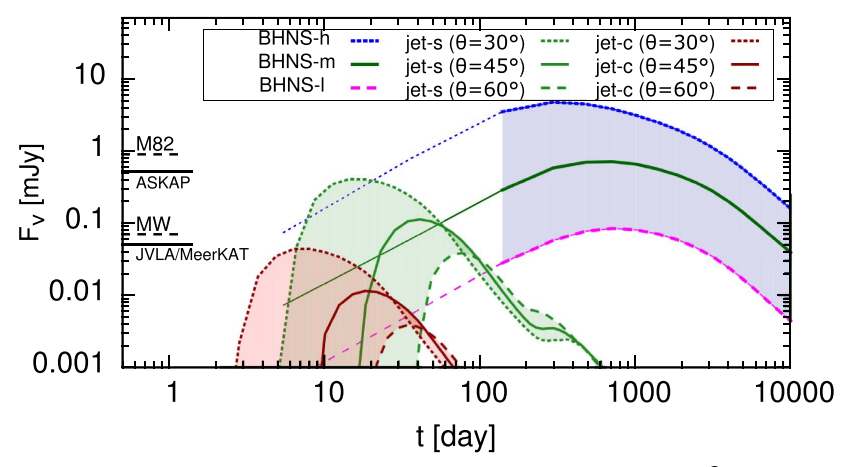

BH-NS, $150 \mathrm{MHz}, \mathrm{D}=300 \mathrm{Mpc}, \mathrm{n}=0.1 \mathrm{~cm}^{-3}$

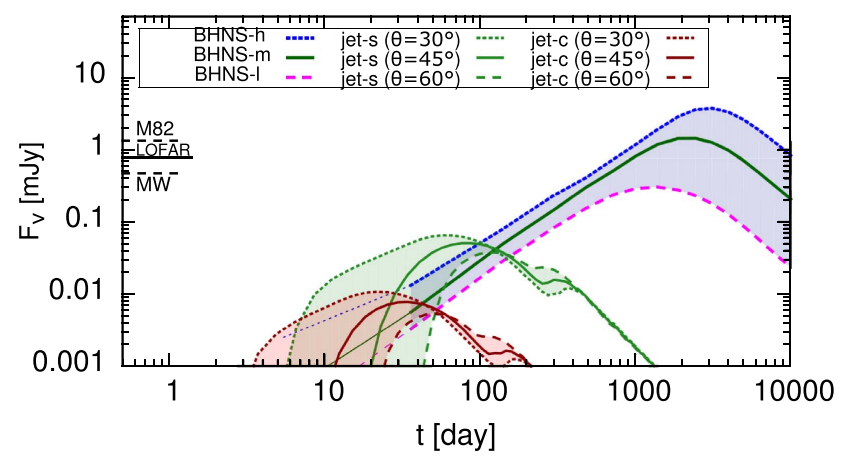

Figure 1. Expected radio light curves at $1.4 \mathrm{GHz}$ (upper panels) and $150 \mathrm{MHz}$ (lower panels) of a DNS merger at $200 \mathrm{Mpc}$ (left panels) and a BH-NS merger at $300 \mathrm{Mpc}$ (right panels). The circum-merger density is set to be $0.1 \mathrm{~cm}^{-3}$. The blue, green, and magenta curves in the blue shaded region correspond to the DNS $\mathrm{h}_{\mathrm{h}}$, $\mathrm{DNS}_{\mathrm{m}}$, and DNS $\mathrm{D}_{1}$ models, respectively. Also shown are the orphan radio afterglows assuming a canonical-jet (red shaded region: jet-c) and a strong-jet (green shaded region: jet-s) with viewing angles of $30^{\circ}$ (dotted), $45^{\circ}$ (solid), and $60^{\circ}$ (dashed). The horizontal solid bars represent the detection limits (7 $\sigma$ noise rms with integration of one hour) of the different radio facilities. The radio flux densities of the galaxies, M82 and the Milky Way, are shown as the horizontal dashed bars assuming a distance of $200 \mathrm{Mpc}$ for DNS and of $300 \mathrm{Mpc}$ for BH-NS systems. For the Milky Way, the peak flux density in the edge-on case for an angular resolution of 7" is shown (see Section 5.1).

(6) Observing the decline in long-lasting radio remnants at $\gtrsim 1000$ days.

The last epoch will naturally only be required if the long-lasting radio remnant candidates are detected in former epochs. By "radio detection," we require, in what follows, a $7 \sigma$ or greater detection during at least one epoch, which corresponds to at least a $40 \%$ change in flux above a $5 \sigma$ noise limit that is required to claim detections. With this detection criterion, most radio variables will be rejected as false positives (see Section 5.2 for details) and this depends on the nuclear versus non-nuclear location of the source. We note that Gaussian thermal fluctuations in the noise are dependent on the number of synthesized beams.

Note that the above recommendation of observations with five survey epochs is for surveys at $1.4 \mathrm{GHz}$. At $150 \mathrm{MHz}$, the epochs (i), (ii), and (iii) are not relevant because, due to the strong synchrotron self-absorption, both the orphan afterglows and radio remnants are too faint to be detected at these epochs and the peak time of the radio remnants is later.

\subsection{Discussion on Circum-merger Densities}

A central concern about the brightness and timescales in the light curves of radio counterparts is the ambiguity in circummerger densities, which can spread over many orders of magnitude. Here we try to address the question of how likely it is that a merger takes place in a relatively high circum-merger density of $\gtrsim 0.1 \mathrm{~cm}^{-3}$. In what follows, we consider this problem using our knowledge of the Galactic DNS population and sGRB afterglow observations.

Double neutron star population in the Galaxy. The interstellar medium (ISM) is known to have highly inhomogeneous structures. The Galactic disk of the Milky Way is filled with three types of gas (Draine 2011): (i) warm neutral medium $\left(f_{V} \sim 0.4, n \sim 0.6 \mathrm{~cm}^{-3}\right)$, (ii) warm ionized medium $\left(f_{V} \sim 0.1, n \sim 0.3-10^{4} \mathrm{~cm}^{-3}\right)$, and (iii) hot ionized medium $\left(f_{V} \sim 0.5, n \sim 0.004 \mathrm{~cm}^{-3}\right)$, where $f_{V}$ is a volume filling factor. Assuming that the Milky Way is typical of galaxies hosting merger events ${ }^{7}$, we estimate that half the volume of the Galactic disk is filled by the ISM with densities of $\gtrsim 0.3 \mathrm{~cm}^{-3}$.

The probability that a merger takes place in the Galactic disk (assuming a half thickness of $\sim 250 \mathrm{pc}$ ) can be estimated based on the spatial distribution of the known Galactic DNS systems. It is worth noting that most of these systems that will coalesce within a Hubble time are located in the Galactic disk even though their characteristic ages are $\gtrsim 100 \mathrm{Myr}$ (e.g., Lorimer 2008). In particular, PSR J0737-3039A/B, PSR B1913+16, and PSR B1906+0746, which are the known DNS systems with the shortest merger times, are located within $300 \mathrm{pc}$ above the Galactic plane. The measured proper motions are 10 and $75 \mathrm{~km} \mathrm{~s}^{-1}$ for PSR J0737-3039A/B and

\footnotetext{
7 The volume filling factor of each phase depends on the supernova rate and the mean ISM density. Li et al. (2015) show that the volume filling factor of the hot ionized medium decreases with the star formation rate density, suggesting that the chance that a merger takes place in a larger ISM density is higher for galaxies with higher star formation rate densities.
} 
DNS, $1.4 \mathrm{GHz}, \mathrm{D}=200 \mathrm{Mpc}$

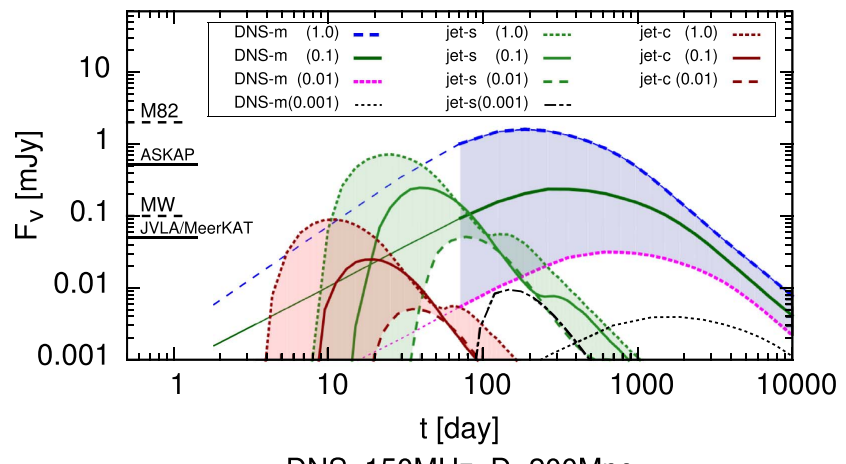

DNS, $150 \mathrm{MHz}, \mathrm{D}=200 \mathrm{Mpc}$

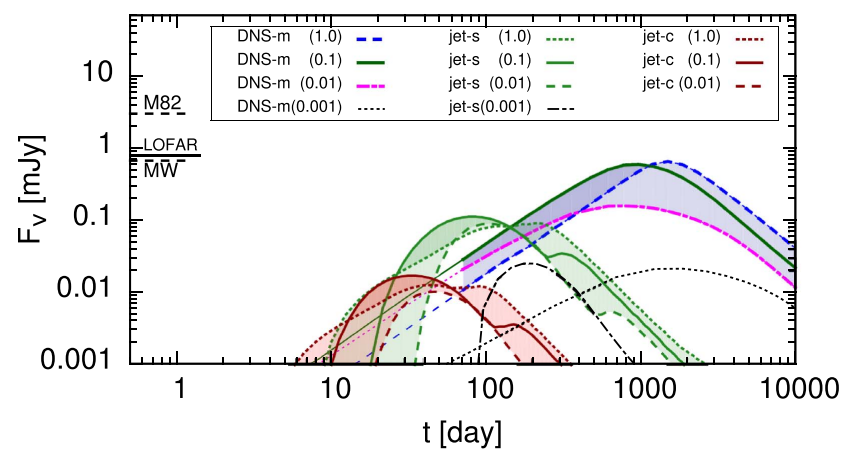

BH-NS, $1.4 \mathrm{GHz}, \mathrm{D}=300 \mathrm{Mpc}$

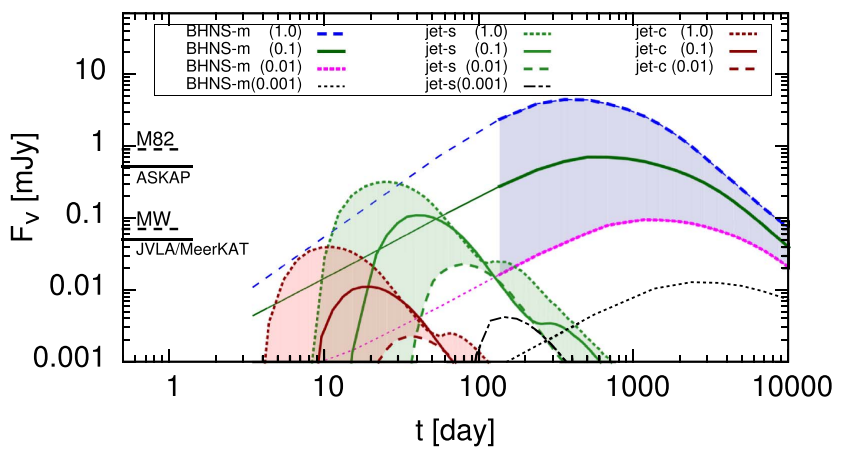

$\mathrm{BH}-\mathrm{NS}, 150 \mathrm{MHz}, \mathrm{D}=300 \mathrm{Mpc}$

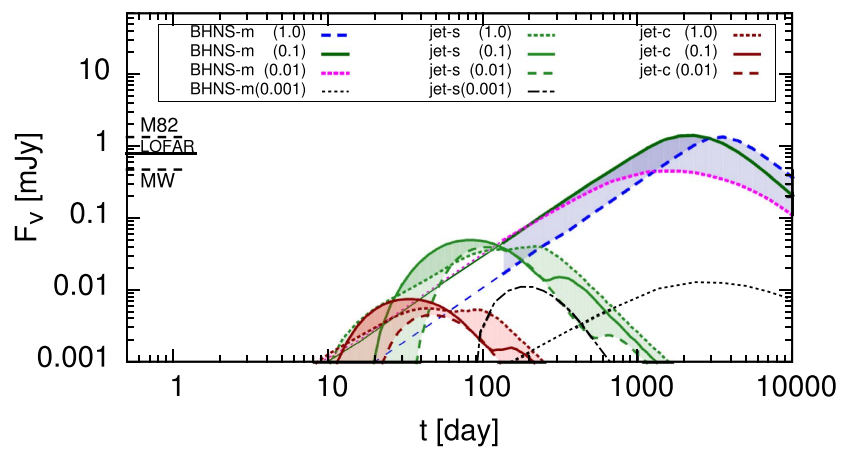

Figure 2. Same as Figure 1 but showing the dependence of the light curves on the circum-merger densities. In the parentheses, circum-merger densities in units of $\mathrm{cm}^{-3}$ are shown. Here we show the light curves for $\mathrm{DNS}_{\mathrm{m}}$ (left), BH-NS $\mathrm{m}_{\mathrm{m}}$ (right), canonical-jet and strong-jet afterglows with a viewing angle of $45^{\circ}$.

PSR B1913+16 respectively (e.g., Weisberg et al. 2010; Beniamini \& Piran 2016), which indicate that these systems have vertical oscillations in the Galactic disk. Thus we expect that they will be in the Galactic disk when they will coalesce. While there are selection biases of pulsar surveys in the Galactic latitude, at least these systems, which contribute predominantly to merger rate estimates calibrated by these known DNS systems, will merge in the Galactic disk.

Also, while incorporating the binary's velocity relative to the Galaxy, the fraction of DNS mergers taking place in the Galactic disk can be estimated based on binary population synthesis studies (Voss \& Tauris 2003; Belczynski et al. 2006; Kiel et al. 2010). Kiel et al. (2010) found that the scale height of DNS mergers is $\sim 500 \mathrm{pc}$, which implies that about $40 \%$ of the mergers are in the Galactic disk. The expected circummerger density is $n \gtrsim 0.3 \mathrm{~cm}^{-3}$ for $\sim 20 \%$ of the mergers and $n \gtrsim 0.1 \mathrm{~cm}^{-3}$ for roughly half of them.

$s G R B$ afterglows. sGRB afterglow observations allow for circum-merger density constraints (e.g., Fong et al. 2015). However, good constraints are necessary from detections of sGRB afterglows because there exists a degeneracy between the density and $\epsilon_{B}$. Indeed, even for a well observed sGRB $130603 \mathrm{~B}$, the range of the estimated densities are $4.9 \cdot 10^{-3}-30 \mathrm{~cm}^{-3}$ (Fong et al. 2014). Fong et al. (2015) show that circum-burst densities span several orders of magnitude, with the median densities being $\approx 3-15 \times 10^{-3} \mathrm{~cm}^{-3}$ under the assumption of $\epsilon_{e}=0.1$ and $\epsilon_{B}=0.01$ or 0.1 . The fraction of sGRBs with densities of $\gtrsim 0.1 \mathrm{~cm}^{-3}$ are $15 \%$ for $\epsilon_{B}=0.1$ and $40 \%$ for $\epsilon_{B}=0.01$. For a subset of bursts for which the observed X-ray frequencies are larger than the cooling frequencies, the circum-burst densities are relatively well constrained, with large median densities of $\approx 0.04-1 \mathrm{~cm}^{-3}$. Within uncertainties in $\epsilon_{B}, \epsilon_{e}$ and the cooling frequencies, we consider that the densities derived from sGRB afterglows are broadly consistent with those estimated from the Galactic DNS distribution.

\section{RADIO DETECTABILITY OF GW MERGER EVENTS}

We now consider the likelihood of finding a radio counterpart to a GW event. Our approach is the following. We assume the GW event to have been detectable by a given network of GW detectors, and we consider different network configurations. We assume that the GW network will have localized the GW event to some area on the sky, which could be as small as a few tens of square degrees but which could plausibly be several hundred square degrees in the immediate future. We assume that the total amount of observing time available for a radio telescope to carry out a search for a counterpart to an individual GW event is $30 \mathrm{hr}$. This "survey allocation" is adopted based on a combination of how much time current radio telescopes tend to allocate to similar efforts and our estimate of the importance of finding radio counterparts. As will become evident below, if the "survey allocation" is longer, it will be more likely to find radio counterparts; if it is shorter, it will be more difficult.

\subsection{The Sensitivity of the Radio Facilities}

From the radiometer equation, the minimum detectable flux density for a radio source is:

$$
S_{\min }=\frac{m \text { SEFD }}{\eta_{c} \sqrt{2 \Delta \nu \Delta t}},
$$


Table 3

Mapping Speed

\begin{tabular}{lcccc}
\hline \hline Parameter & LOFAR & JVLA & ASKAP & MeerKAT \\
\hline Frequency (GHz) & 0.150 & 1.4 & 1.4 & 1.4 \\
SEFD (Jy) & 31 & 13 & 87 & 7.7 \\
FOV (deg ${ }^{2}$ ) & 11.35 & 0.25 & 30 & 0.86 \\
Bandwidth (MHz) & 90 & 600 & 270 & 690 \\
Survey Speed $\left(\mathrm{deg}^{2} \mathrm{hr}^{-1}\right)$ & $8.2(240)$ & 14 & 20 & 140 \\
Angular resolution $\left(\mathrm{arcsec}^{-1}\right)$ & 10 & 4.3 & 7 & 5.25 \\
\hline
\end{tabular}

Note. We have assumed a standard correlator quantization loss of 0.9. Note that the values of the JVLA are based on real results while the other values are based on current predictions of performance. The bandwidths are the values after taking the loss due to RFI into account. Here the survey speeds at $1 \sigma$ noise rms of $0.1 \mathrm{mJy}$ are shown but the one of $0.7 \mathrm{mJy}$ is also shown in the parentheses for the LOFAR since flux densities at $150 \mathrm{MHz}$ of optically thin sources are brighter than those at $1.4 \mathrm{GHz}$ by a factor of $\sim 7$. The JVLA B configuration using the natural weighting and HBA-Inner Dual configuration of the LOFAR using 40 stations are chosen.

where SEFD is the system equivalent flux density or the flux density that a source would have in order to be equivalent to the noise power in the system, $\eta_{c}$ is an efficiency factor accounting for losses during correlation, $\Delta \nu$ is the processed bandwidth for the observations, and $\Delta t$ is the total integration time for a given field. It is assumed that the noise in the image is Gaussian so that a source can be detected if it is a factor of $m$ stronger than the rms noise level (i.e., $m-\sigma$ ). Formally, Equation (9) specifies the thermal noise limit; in practice, a variety of factors, such as dynamic range limitations due to calibration errors and low-level radio frequency interference may all contribute to a larger image noise level. As long as the instrument is well understood, the resulting point-spread function of incomplete uv-coverage can be completely deconvolved away.

Also relevant for searches is the telescope's field-of-view (FOV). Traditionally, this value has been taken to be the halfpower pattern of the individual element in the array, which itself has a circular aperture,

$$
\Omega \approx \frac{\pi}{4}\left(1.2 \frac{\lambda}{D}\right)^{2},
$$

for a dish antenna of diameter $D$ observing at a wavelength $\lambda$. Emerging technologies in which the electric field is sampled at the focal plane of the antenna, with "phased array feeds," offer the potential of much larger FOVs. Equation (10) is the FOV for a single pointing direction. Surveying requires careful attention to the placement of multiple FOVs to ensure that the sensitivity across the entire region to be surveyed is approximately constant. For instance, for a dish antenna-based array, surveys are typically designed so that the spacing between adjacent pointing centers is approximately half the nominal beamwidth. The spacing between adjacent pointings is $\sqrt{2}$ or $\sqrt{3}$ for a survey with uniform sensitivity. The survey speed for a given sensitivity is:

$$
\dot{\Omega} \approx \frac{\Omega}{2 \Delta t} .
$$

Table 3 summarizes relevant values for a number of telescopes expected to be operational in the latter half of this decade, when a number of ground-based GW detectors are also coming online. Here we consider Jansky VLA (Perley et al. 2011), ASKAP (Johnston et al. 2008), and MeerKAT (Booth \& Jonas 2012) at the $\mathrm{GHz}$ band. We also consider the detectability at $150 \mathrm{MHz}$ with the LOFAR (van Haarlem et al. 2013). Note that, apart from these telescopes, there are other relevant telescopes including: WSRT/Apertif (Oosterloo et al. 2010), GMRT (Intema et al. 2016), and MWA (Tingay et al. 2013). Note that the values of the JVLA are based on current data while the other values are based on current predictions of performance.

\subsection{Detectability for Networks of 3-5 GW Detectors}

In what follows, we investigate the detectability of the radio counterparts of GW merger events taking into account the distances, inclinations and sky localization errors of GW detections (Section 2.3), the radio light curves (Section 3.3), and the survey speeds of current and future radio facilities (Section 4.1). As described in Section 2.3, we simulate GW parameter errors and compute a diversity of radio light curves for each GW-detectable merger. Given the large uncertainty in estimates of circum-merger densities from afterglow modelings as discussed in Section 3.4, we choose relatively high circummerger densities of $1,0.1$, and $0.01 \mathrm{~cm}^{-3}$ based on the Galactic DNS population. In what follows, we assume the microphysics parameters $\epsilon_{e}=\epsilon_{b}=0.1$ and $p=2.5$.

As discussed in Section 3.3, we assume in our simulations that each GW-detectable merger is observable in five observation epochs approximately spaced by logarithmic time intervals and with a $30 \mathrm{hr}$ total observation time in each epoch. Critically, we have not taken into account Northern and Southern hemisphere considerations of the GW sky localizations, and hence the following relative detectability fractions should be reduced by approximately a factor of two. Table 4 lists the derived radio-GW detection likelihood of each model for each radio facility. For DNS mergers with $n=1 \mathrm{~cm}^{-3}$, the majority of $\mathrm{GW}$ events for $\mathrm{DNS}_{\mathrm{h}}$ and $\mathrm{DNS}_{\mathrm{m}}$ will be detectable in the $\mathrm{GHz}$ band. Unsurprisingly as shown in Equation (3) and Table 2, we find that the radio detection likelihood for GW mergers decreases as the density decreases, e.g., for $\mathrm{DNS}_{\mathrm{m}}$, $20 \%-60 \%$ for $0.1 \mathrm{~cm}^{-3}$ and $3 \%-10 \%$ for $0.01 \mathrm{~cm}^{-3}$. For $\mathrm{BH}-$ NS cases, similar results exist because we expect an increase in the intrinsic higher radio luminosity (Section 3.2). For orphan afterglows with $n \gtrsim 0.1 \mathrm{~cm}^{-3}, 30 \%-90 \%$ of the events will be detectable for those with strong-jets and 5\%-30\% for those with canonical-jets.

We now discuss further details in how to optimize our search in detecting radio-GW mergers. Figures 3 and 4 show the maximum flux density at $1.4 \mathrm{GHz}$ and $150 \mathrm{MHz}$ for each event among the five epochs as a function of the $2 \sigma \mathrm{GW}$ localization areas using GW Net 3. The filled points represent the nearby events at distances of $<200 \mathrm{Mpc}$. The diagonal lines show the $7 \sigma$ noise rms of the radio facilities corresponding to the detection threshold. As expected, we find that the detectability of the well localized GW events, which occur more often than not at nearby distances, is much higher than that of the poorly localized ones. The detection likelihood roughly behaves as $\propto \Delta \Omega_{\mathrm{GW}}^{-1.5}$, where $\Delta \Omega_{\mathrm{GW}}$ is the GW solid angle measure on the sky. Furthermore, the integration time of each FOV is longer for such events. For instance, for $\mathrm{DNS}_{m}$ with $n=0.1 \mathrm{~cm}^{-3}$, the JVLA detects more than $60 \%$ of events localized within a sky 
Table 4

Radio-GW Detection Likelihood (\%) for Each Radio Telescope and GW Net 3 (Shown in Parentheses Are GW Net 5)

\begin{tabular}{|c|c|c|c|c|c|c|c|}
\hline Model & $n\left(\mathrm{~cm}^{-3}\right)$ & JVLA (1.4 GHz) & JVLA (3 GHz) & ASKAP & MeerKAT & LOFAR & Host \\
\hline $\mathrm{DNS}_{\mathrm{h}}$ & 1.0 & $100(100)$ & $100(100)$ & $100(100)$ & $100(100)$ & $51(52)$ & B \\
\hline $\mathrm{DNS}_{\mathrm{m}}$ & 1.0 & $79(88)$ & $72(78)$ & 87 (93) & 99 (99) & 37 (39) & M \\
\hline $\mathrm{DNS}_{1}$ & 1.0 & $21(32)$ & $13(20)$ & $24(21)$ & 64 (71) & 19 (19) & M \\
\hline $\mathrm{BH}-\mathrm{NS}_{\mathrm{h}}$ & 1.0 & $100(100)$ & $100(100)$ & $100(100)$ & $100(100)$ & $30(30)$ & B \\
\hline $\mathrm{BH}-\mathrm{NS}_{\mathrm{m}}$ & 1.0 & $98(96)$ & $94(93)$ & 98 (97) & $100(100)$ & $20(21)$ & B \\
\hline $\mathrm{BH}-\mathrm{NS}_{1}$ & 1.0 & $41(43)$ & $34(34)$ & $45(38)$ & $74(82)$ & $17(11)$ & M \\
\hline strong-jet & 1.0 & $49(65)$ & $58(68)$ & $53(55)$ & $86(86)$ & $8(3)$ & M \\
\hline canonical-jet & 1.0 & $11(13)$ & $10(14)$ & $8(6)$ & $27(31)$ & $0(0)$ & $\mathrm{F}$ \\
\hline $\mathrm{DNS}_{\mathrm{h}}$ & 0.1 & $86(93)$ & $73(79)$ & $91(95)$ & $100(99)$ & $78(86)$ & B \\
\hline $\mathrm{DNS}_{\mathrm{m}}$ & 0.1 & $21(31)$ & $13(19)$ & $21(21)$ & $62(67)$ & $44(46)$ & M \\
\hline $\mathrm{DNS}_{1}$ & 0.1 & $6(4)$ & $3(3)$ & $3(2)$ & $12(15)$ & $10(8)$ & $\mathrm{F}$ \\
\hline $\mathrm{BH}-\mathrm{NS}_{\mathrm{h}}$ & 0.1 & 98 (97) & $93(93)$ & 99 (98) & $100(100)$ & $55(54)$ & B \\
\hline $\mathrm{BH}-\mathrm{NS}_{\mathrm{m}}$ & 0.1 & 44 (44) & $35(36)$ & $47(41)$ & 77 (83) & $42(43)$ & M \\
\hline $\mathrm{BH}-\mathrm{NS}_{1}$ & 0.1 & $4(6)$ & $2(4)$ & $3(2)$ & $21(27)$ & $19(18)$ & M \\
\hline strong-jet & 0.1 & $36(41)$ & $35(39)$ & $37(34)$ & $55(62)$ & $9(6)$ & M \\
\hline canonical-jet & 0.1 & $8(8)$ & $8(7)$ & 7 (4) & $20(19)$ & $2(1)$ & $\mathrm{F}$ \\
\hline $\mathrm{DNS}_{\mathrm{h}}$ & 0.01 & $20(26)$ & $13(16)$ & $21(15)$ & $60(59)$ & $61(64)$ & M \\
\hline $\mathrm{DNS}_{\mathrm{m}}$ & 0.01 & $4(4)$ & $2(3)$ & $3(2)$ & $12(11)$ & $13(11)$ & $\mathrm{F}$ \\
\hline $\mathrm{DNS}_{1}$ & 0.01 & $0(1)$ & $0(0)$ & $0(0)$ & $2(3)$ & $2(1)$ & $\mathrm{F}$ \\
\hline $\mathrm{BH}-\mathrm{NS}_{\mathrm{h}}$ & 0.01 & 41 (43) & 34 (34) & $45(38)$ & $74(82)$ & $67(70)$ & M \\
\hline $\mathrm{BH}-\mathrm{NS}_{\mathrm{m}}$ & 0.01 & $7(8)$ & $3(4)$ & $4(2)$ & $23(28)$ & 28 (29) & M \\
\hline $\mathrm{BH}-\mathrm{NS}_{1}$ & 0.01 & $1(1)$ & $1(1)$ & $1(1)$ & $1(2)$ & $1(2)$ & $\mathrm{F}$ \\
\hline strong-jet & 0.01 & 15 (19) & $12(18)$ & $15(17)$ & $29(34)$ & $10(6)$ & $\mathrm{F}$ \\
\hline canonical-jet & 0.01 & $3(4)$ & 3 (4) & $1(1)$ & $10(8)$ & $1(1)$ & $\mathrm{F}$ \\
\hline
\end{tabular}

Note. Here the detection requires at least a $7 \sigma$ detection during at least one observation epoch (10 day, 30 day, 100 day, 300 day, or 1000 day after GW detections). A total observation time of $30 \mathrm{hr}$ is assumed in each observation epoch. In the last column, the comparison of the radio counterpart with the contamination of the hosts is shown. B: brighter than $10^{29} \mathrm{erg} \mathrm{s}^{-1} \mathrm{~Hz}^{-1}$, F: fainter than $5 \cdot 10^{27} \mathrm{erg} \mathrm{s}^{-1} \mathrm{~Hz}^{-1} \mathrm{M}$ : between B and F. Note that Northern and Southern hemisphere considerations of the GW sky localizations are not taken into account, and hence the relative detectability fractions should be reduced by approximately a factor of two.

area of $20 \mathrm{deg}^{2}$. In contrast, the detection likelihood is less than $10 \%$ for the poorly localized events with $\gtrsim 100 \mathrm{deg}^{2}$.

For a given detection likelihood, localization area of a GW event, and radio luminosity, one can set an optimized survey allocation time $T$ based on Figures 3-6. For instance, in order to achieve a detection likelihood of $\sim 50 \%$ for GW Net 3 (Net 5 ), the survey allocation time can be estimated as

$$
T \sim\left\{\begin{aligned}
4(60) & \mathrm{hr}\left(\frac{\Delta \Omega_{\mathrm{GW}}}{10 \mathrm{deg}^{2}}\right)^{3}\left(\frac{\dot{\Omega}}{14 \mathrm{deg}^{2} \mathrm{hr}^{-1}}\right)^{-1} \\
& \times\left(\frac{L_{\nu}}{10^{28} \mathrm{erg} \mathrm{s}^{-1} \mathrm{~Hz}^{-1}}\right)^{-2} \quad\left(\Delta \Omega_{\mathrm{GW}} \leqslant \Omega_{c}\right), \\
35(60) \mathrm{hr}\left(\frac{\Delta \Omega_{\mathrm{GW}}}{10 \operatorname{deg}^{2}}\right)\left(\frac{\Omega}{14 \operatorname{deg}^{2} \mathrm{hr}^{-1}}\right)^{-1} & \times\left(\frac{L_{\nu}}{10^{28} \mathrm{erg} \mathrm{s}^{-1} \mathrm{~Hz}^{-1}}\right)^{-2} \quad\left(\Delta \Omega_{\mathrm{GW}}>\Omega_{c}\right),
\end{aligned}\right.
$$

where $\dot{\Omega}$ is the survey speed at $1 \sigma \mathrm{rms}$ noise of $0.1 \mathrm{mJy}$ and $\Omega_{c} \sim 30(10) \mathrm{deg}^{2}$. This estimation is valid only in the case of $\Delta \Omega_{\mathrm{GW}}>\Omega_{\mathrm{FOV}}$. Note that $T$ is rather sensitive to the radio luminosity of the source.

Figure 5 shows the results of the detectability of orphan radio afterglows. The scatter in the maximum flux densities is much larger than the corresponding case for the long-lasting radio remnants due to the viewing angle effects. Note that the dependence of the detection likelihood on the circum-merger density is somewhat weaker than that of the long-lasting remnants in this density range.
Figure 6 shows the same cases as before though this time in the instance of GW Net 5. The detection likelihood does not change significantly because the gain in the radio sensitivity due to better localizations compensates with the loss in the radio flux brightness due to the increase of the $\mathrm{GW}$ detectable distances.

The detectability at $150 \mathrm{MHz}$ is limited by the confusion noise $(\sim 0.6 \mathrm{mJy})$ for the configuration employed in this work. For detecting the radio counterparts by the LOFAR with a similar detection likelihood as those at $\mathrm{GHz}$ band, the confusion noise should be reduced by at least an order of magnitude. Note that, however, the actual confusion limit may be lower than that we use in this work (Heald et al. 2015). Furthermore, it will be reduced by increasing the angular resolution in the near future (see, e.g., Shimwell et al. 2016).

The detection likelihoods that we obtain here change for a different choice of the microphysics parameters. The dependence of the peak flux on those parameters is discussed in Section 3.1. For instance, in the case of $\epsilon_{b}=0.01$, the peak flux densities at $1.4 \mathrm{GHz}$ is lower by an order of magnitude than those with $\epsilon_{b}=0.1$, e.g., the detection likelihood of $\mathrm{DNS}_{\mathrm{m}}$ with $n=1 \mathrm{~cm}^{-3}$ and $\epsilon_{b}=0.01$ by JVLA is $20 \%$. For the orphan afterglows, the detectability depends also on the initial jet half-opening angle. One can expect a higher detection likelihood for a wider jet-half opening angle because the probability that an observer is located within the initial opening angle is higher.

Detectability with two $G W$ detectors and/or use of $G W$ volumes. The advanced LIGO detectors have been operating in 
DNS, Net $3,1.4 \mathrm{GHz}, 30 \mathrm{hr}, 1.0 \mathrm{~cm}^{-3}$

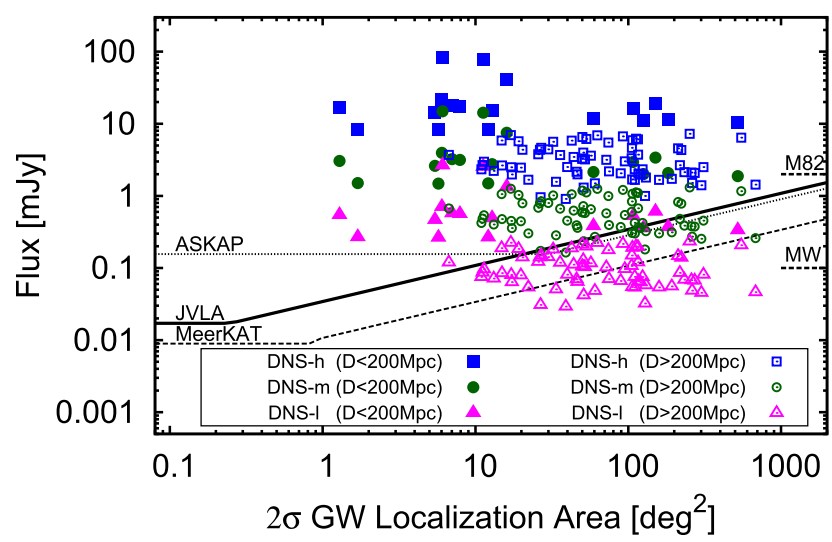

DNS, Net $3,1.4 \mathrm{GHz}, 30 \mathrm{hr}, 0.1 \mathrm{~cm}^{-3}$

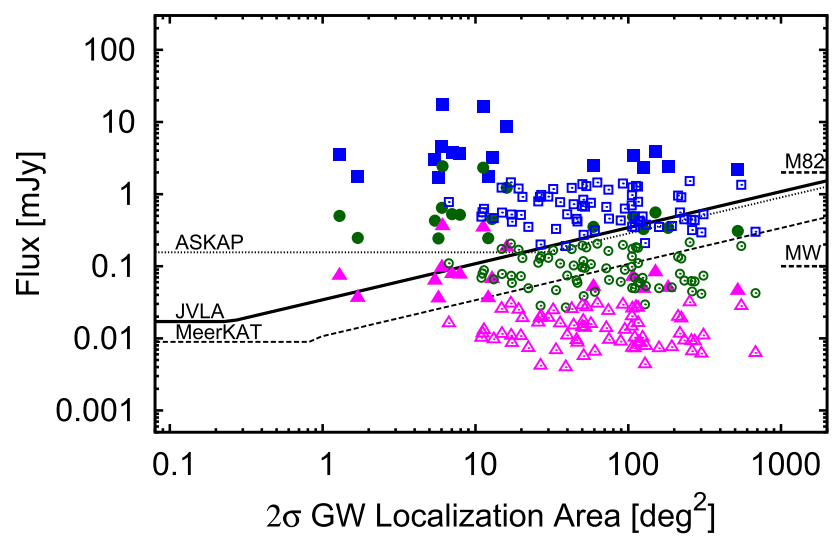

DNS, Net $3,1.4 \mathrm{GHz}, 30 \mathrm{hr}, 0.01 \mathrm{~cm}^{-3}$

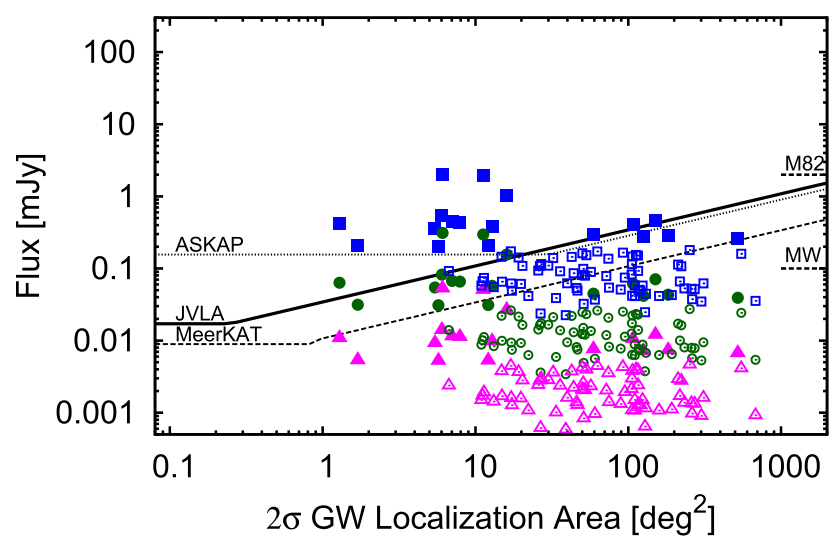

BH-NS, Net $3,1.4 \mathrm{GHz}, 30 \mathrm{hr}, 1.0 \mathrm{~cm}^{-3}$

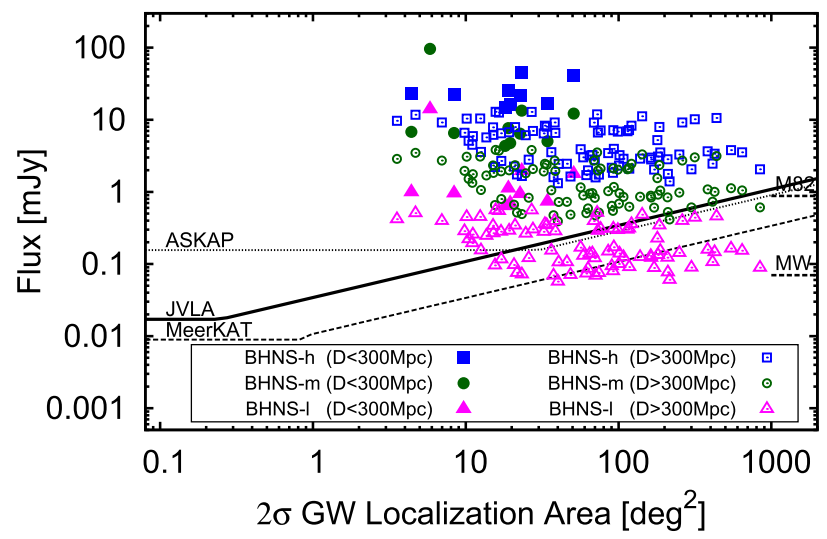

BH-NS, Net $3,1.4 \mathrm{GHz}, 30 \mathrm{hr}, 0.1 \mathrm{~cm}^{-3}$

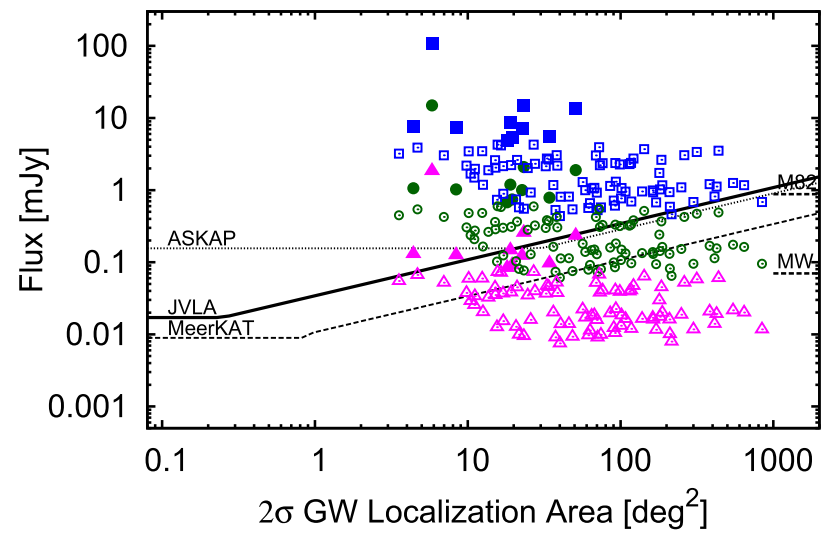

BH-NS, Net $3,1.4 \mathrm{GHz}, 30 \mathrm{hr}, 0.01 \mathrm{~cm}^{-3}$

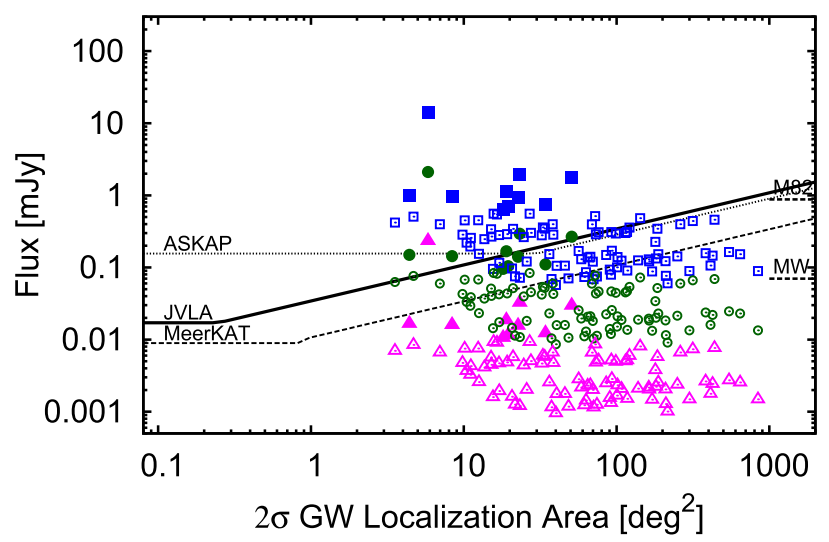

Figure 3. The peak flux densities of long-lasting radio remnants as a function of the $2 \sigma \mathrm{GW}$ localization areas using GW Net 3 for DNS mergers (left panels) and BHNS mergers (right panels). We set the circum-merger densities to be $1.0 \mathrm{~cm}^{-3}$ (upper panels), $0.1 \mathrm{~cm}^{-3}$ (middle panels), and $0.01 \mathrm{~cm}$ (lower panels). The blue filled squares, green filled circles, and red filled triangles show the high, medium, and low ejecta models within a distance of $200 \mathrm{Mpc}$, respectively. The open symbols show those events that occur greater than $200 \mathrm{Mpc}$. The lines show the $7 \sigma$ noise levels of the radio facilities assuming that the total observation time of each epoch is $30 \mathrm{hr}$ with a survey speed given in Section 4.1. As examples, the radio flux densities at $1.4 \mathrm{GHz}$ of the galaxies, M82, and the Milky Way, are shown as the horizontal dashed bars assuming a distance of $200 \mathrm{Mpc}$ in the case of DNS and of $300 \mathrm{Mpc}$ for BH-NS mergers. For the Milky Way, the peak flux density in the edge-on case for an angular resolution of 7" is shown (see Section 5.1). Here Northern and Southern hemisphere considerations of the GW sky localizations are not taken into account, and hence the relative detectability fractions should be reduced by approximately a factor of two.

their first science run (O1) from 2015 September to 2016 January (The LIGO Scientific Collaboration et al. 2013). For their second run, the two detectors will undergo further upgrades and will operate jointly in the second half of 2016 with the first upgraded version of advanced Virgo (The LIGO Scientific Collaboration et al. 2013). We consider radio detectability of GW mergers using only two advanced LIGO detectors (Kasliwal \& Nissanke 2014; Singer et al. 2014). In this case, the GW localization areas will be as large as several hundred to a thousand of $\mathrm{deg}^{2}$ for events at smaller distances of $\lesssim 80 \mathrm{Mpc}$ and $\lesssim 120 \mathrm{Mpc}$ for DNS and BH-NS mergers respectively. In spite of the poor GW localizations, the radio 
DNS, Net $3,150 \mathrm{MHz}, 30 \mathrm{hr}, 1.0 \mathrm{~cm}^{-3}$

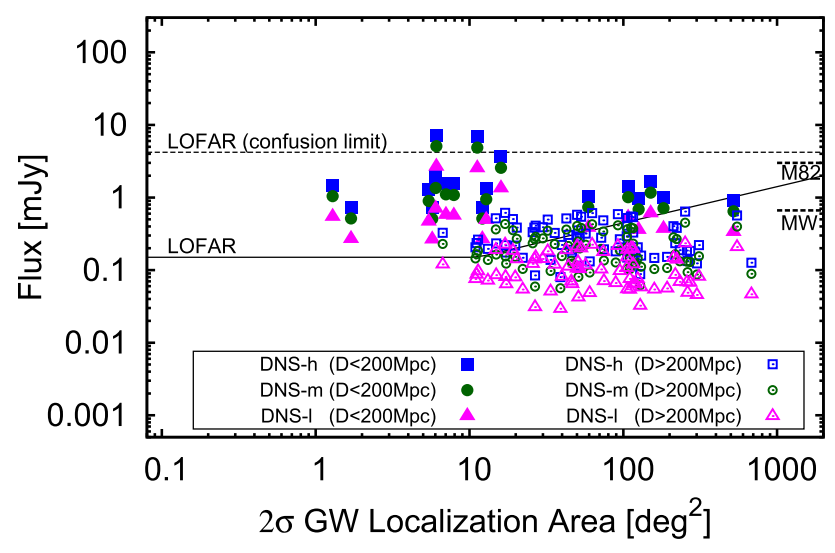

DNS, Net $3,150 \mathrm{MHz}, 30 \mathrm{hr}, 0.1 \mathrm{~cm}^{-3}$

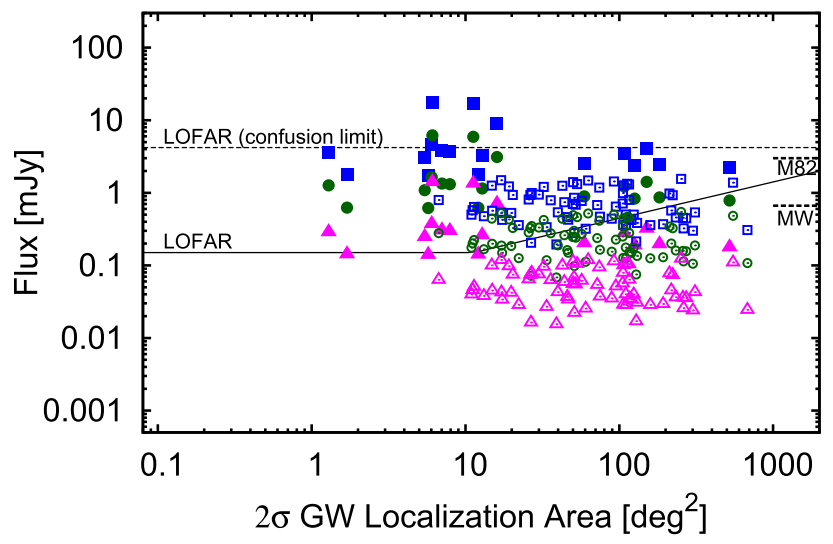

DNS, Net $3,150 \mathrm{MHz}, 30 \mathrm{hr}, 0.01 \mathrm{~cm}^{-3}$

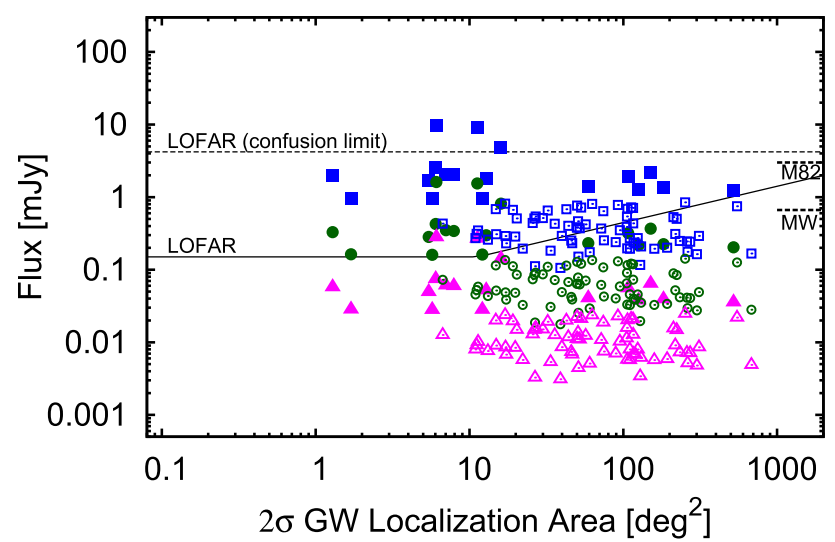

BH-NS, Net 3, 150MHz, 30hr, $1.0 \mathrm{~cm}^{-3}$

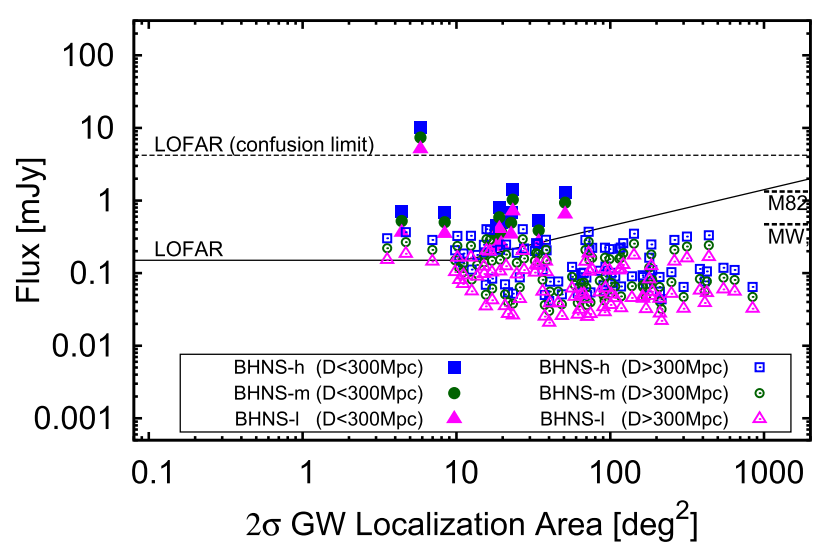

BH-NS, Net 3, $150 \mathrm{MHz}, 30 \mathrm{hr}, 0.1 \mathrm{~cm}^{-3}$

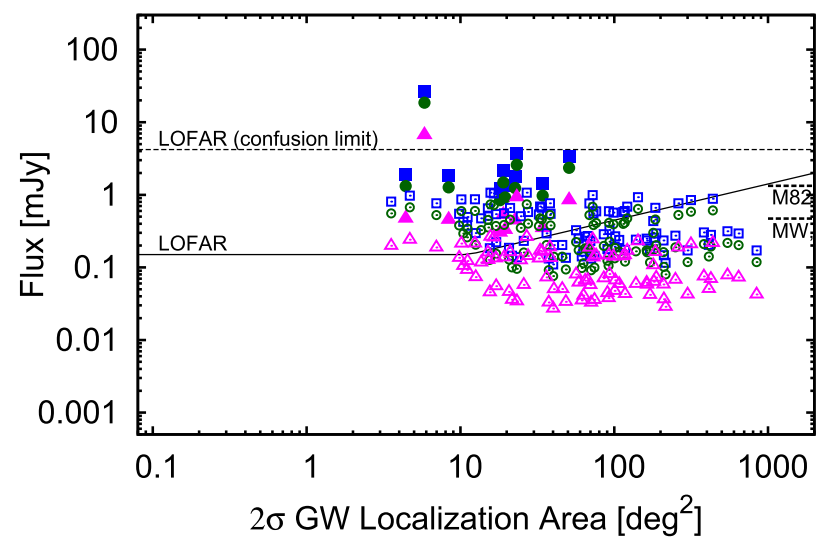

BH-NS, Net 3, 150MHz, 30hr, $0.01 \mathrm{~cm}^{-3}$

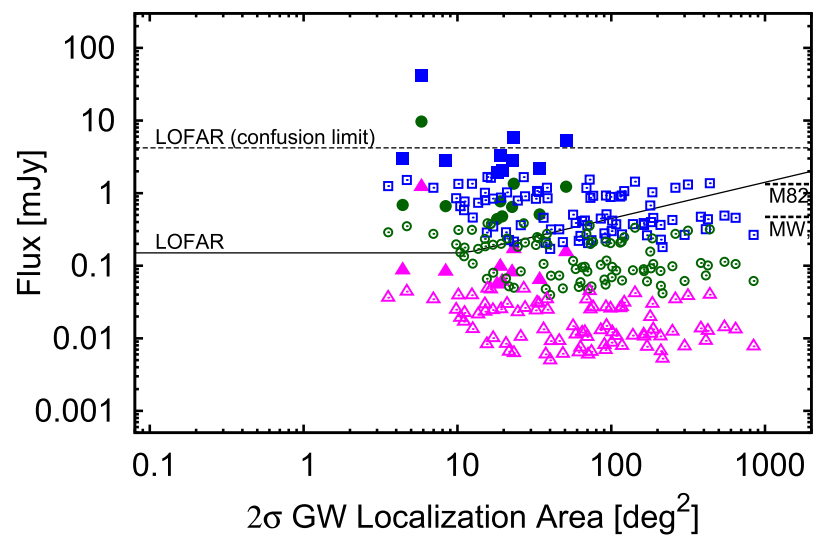

Figure 4. The same as Figure 3 but for $150 \mathrm{MHz}$. The expected $7 \sigma$ detection limit using the confusion limit of the LOFAR with an angular resolution of $10^{\prime \prime}$ is also shown.

detection likelihood is generally higher than the three- and fivedetector networks because of the smaller GW detectable distances, though of course we also expect far fewer merger events in the smaller GW detectable volumes. In particular, for these large GW sky localizations, recent works have shown how the use of $\mathrm{GW}$ volumes, together with local universe Galaxy catalogs (either tracing H-I or $\mathrm{H}-\alpha$ - see e.g., Kasliwal 2011), can provide optimal targeted ranked galaxy follow-up strategies or can substantially reduce the number of astrophysical false-positives using the spatial coincidence within or nearby local galaxies (e.g., NKG13, Gehrels et al.
2016; Singer et al. 2016). For radio follow-up of long-lasting flares and afterglows, we emphasise that the combined use of GW volumes and galaxy catalogs are critical, in particular, for two reasons. First, targeted galaxy follow-up will be optimum when the GW areas cover hundreds of deg. ${ }^{2}$ because of the small FOV relative to the mapping speed of some radio telescopes (Table 3). Second, the GW strain will provide accurate Bayesian-derived distance measures on the days timescale comparable to the timescale for our suggested first observational epoch in the radio; we discuss this in detail in the following Section 5. 
jet (DNS), Net $3,1.4 \mathrm{GHz}, 30 \mathrm{hr}, 1.0 \mathrm{~cm}^{-3}$

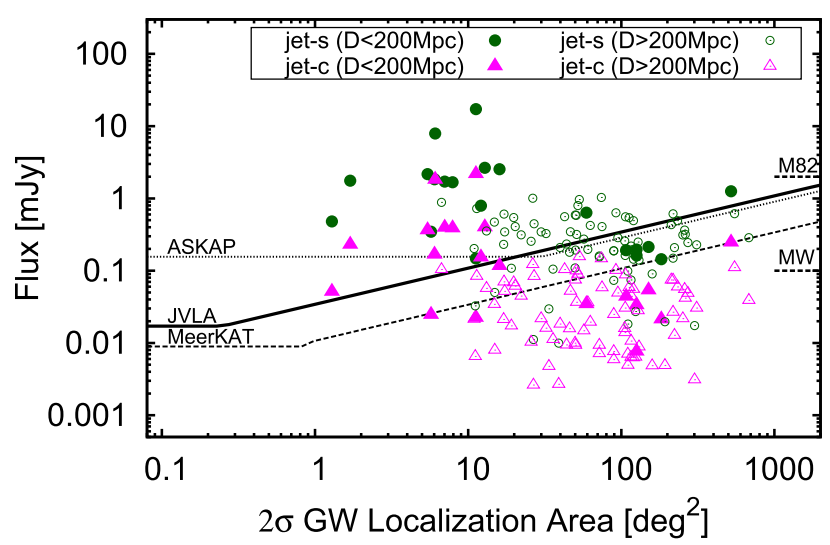

jet (DNS), Net $3,1.4 \mathrm{GHz}, 30 \mathrm{hr}, 0.1 \mathrm{~cm}^{-3}$

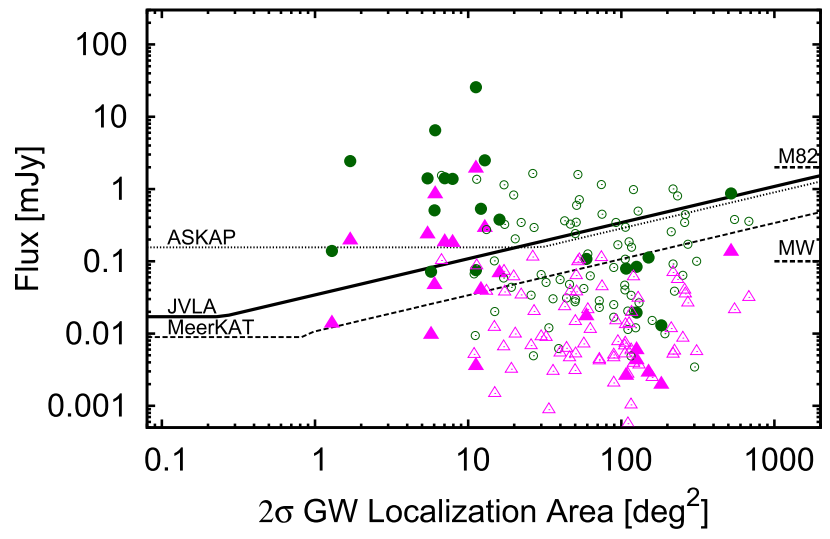

jet (DNS), Net 3, $1.4 \mathrm{GHz}, 30 \mathrm{hr}, 0.01 \mathrm{~cm}^{-3}$

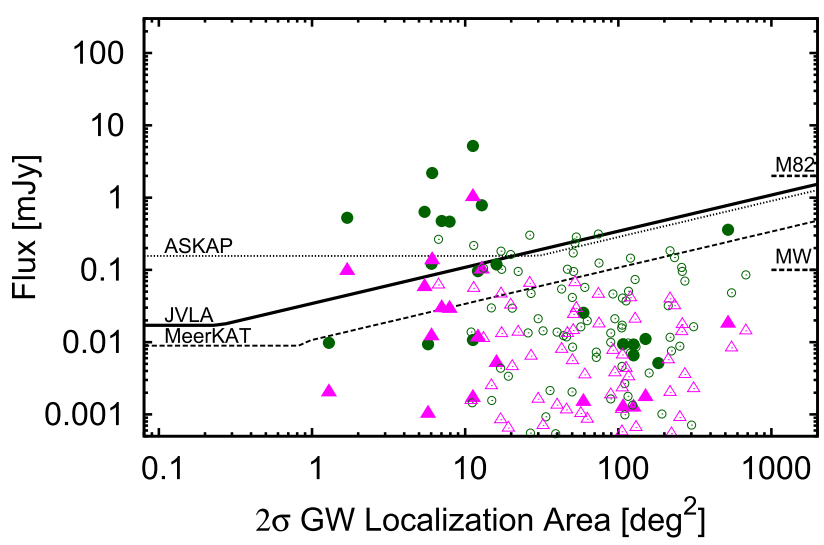

jet (DNS), Net 3, $150 \mathrm{MHz}, 30 \mathrm{hr}, 1.0 \mathrm{~cm}^{-3}$

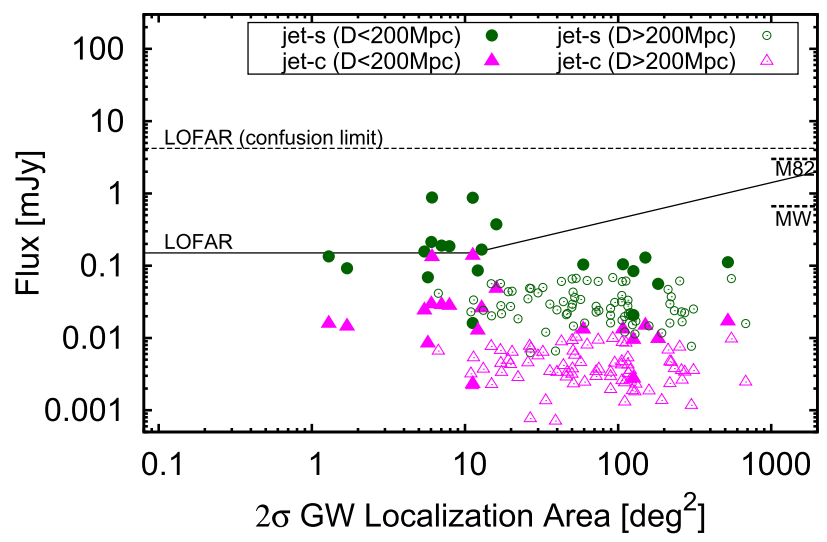

jet (DNS), Net 3, $150 \mathrm{MHz}, 30 \mathrm{hr}, 0.1 \mathrm{~cm}^{-3}$

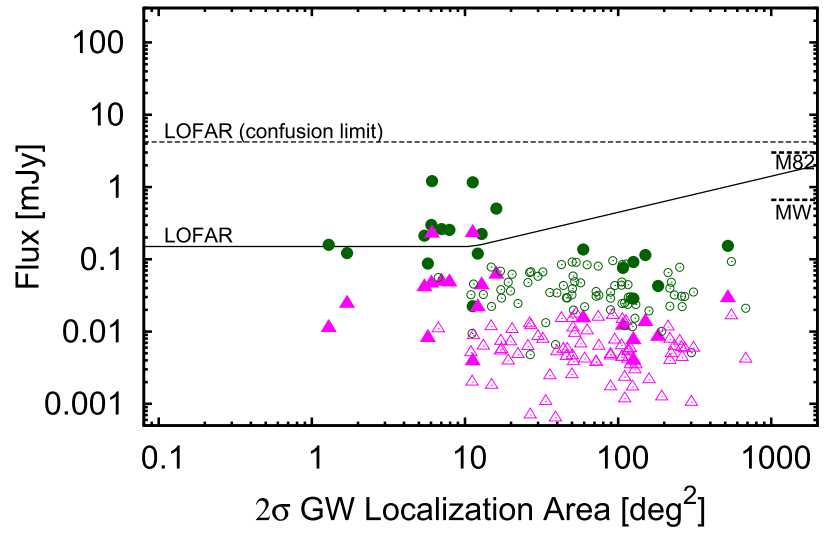

jet (DNS), Net 3, 150MHz, 30hr, $0.01 \mathrm{~cm}^{-3}$

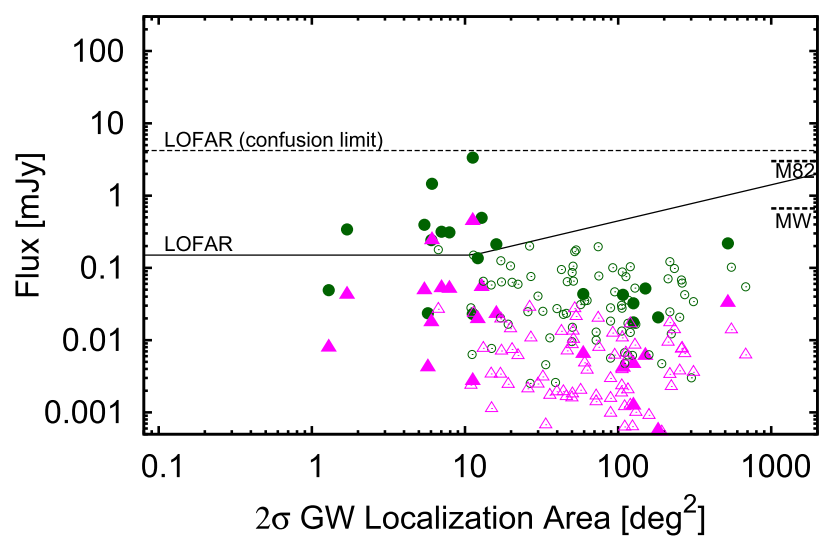

Figure 5. The same as Figure 3 but for the orphan afterglows. Left and right panels show the result of $1.4 \mathrm{GHz}$ and of $150 \mathrm{MHz}$ respectively.

\section{RADIO COUNTERPART IDENTIFICATION}

When estimating the radio detectability of GW mergers in Section 4, we have not taken into account: (i) the radio emission of the host galaxy that may significantly contaminate the merger, and (ii) any astrophysical false-positive transients and variables that may mimic an NS binary merger in the huge swaths of the searched sky. For instance, these transients and variables span a variety of sources from tidal disruption events, different flavours of supernovae, long GRBs to active galactic nuclei (AGNs). In this section we now discuss the challenges posed first by the host galaxy contamination and second by the astrophysical false positive transients. We then provide strategies to overcome them.

\subsection{Host Galaxy Contamination}

The host galaxies of DNS and BH-NS mergers exhibit radio emission, which may contaminate the emission from the radio counterparts of $\mathrm{GW}$ events. For example, the $1.4 \mathrm{GHz}$ radio luminosities of M33, the Milky Way, and M82 are 1027.5, $10^{28.5}$, and $10^{29}$ erg s$^{-1} \mathrm{~Hz}^{-1}$ (Beuermann et al. 1985; Condon 
DNS, Net $5,1.4 \mathrm{GHz}, 30 \mathrm{hr}, 1.0 \mathrm{~cm}^{-3}$

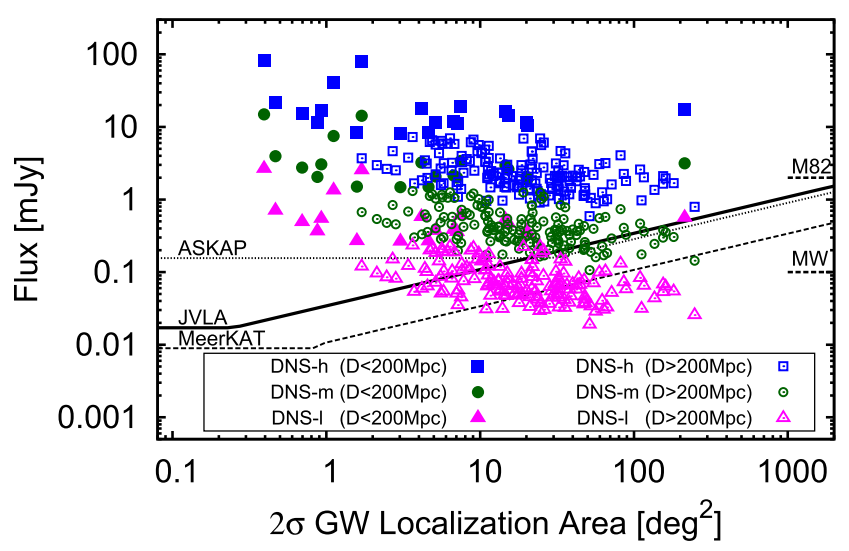

DNS, Net $5,1.4 \mathrm{GHz}, 30 \mathrm{hr}, 0.1 \mathrm{~cm}^{-3}$

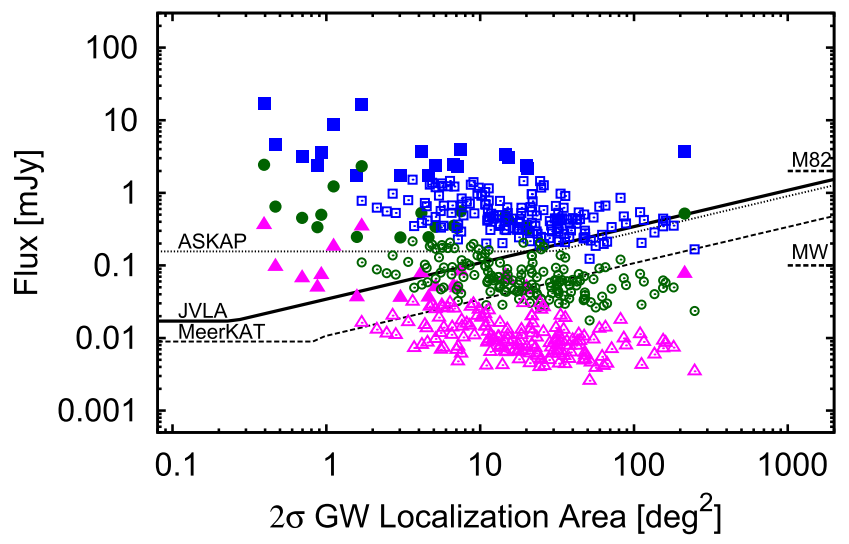

DNS, Net $5,1.4 \mathrm{GHz}, 30 \mathrm{hr}, 0.01 \mathrm{~cm}^{-3}$

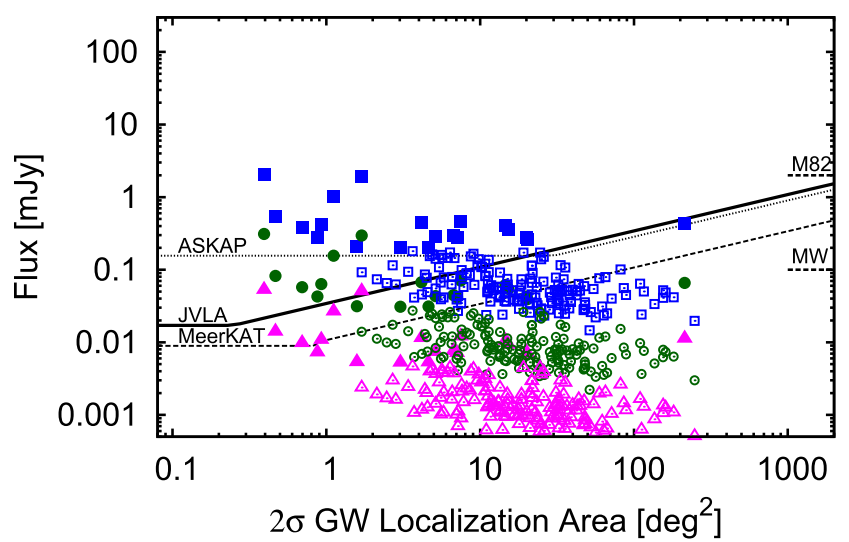

BH-NS, Net $5,1.4 \mathrm{GHz}, 30 \mathrm{hr}, 1.0 \mathrm{~cm}^{-3}$

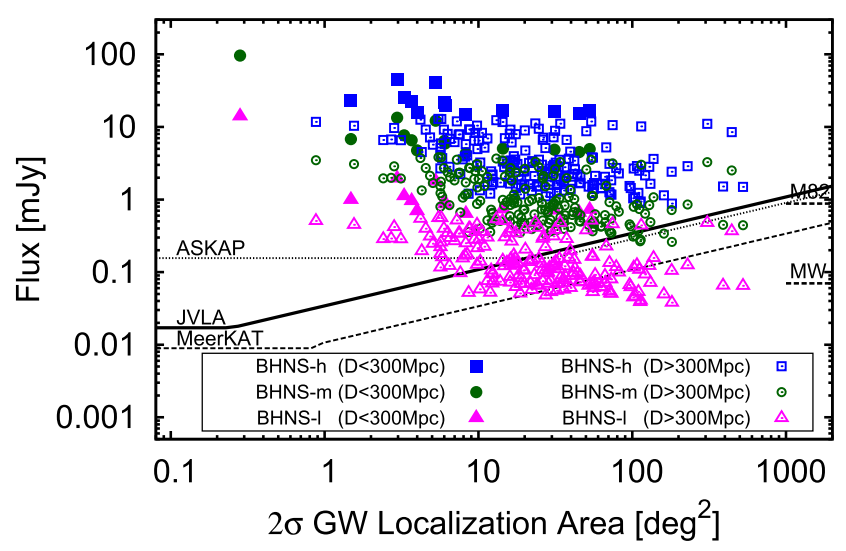

BH-NS, Net $5,1.4 \mathrm{GHz}, 30 \mathrm{hr}, 0.1 \mathrm{~cm}^{-3}$

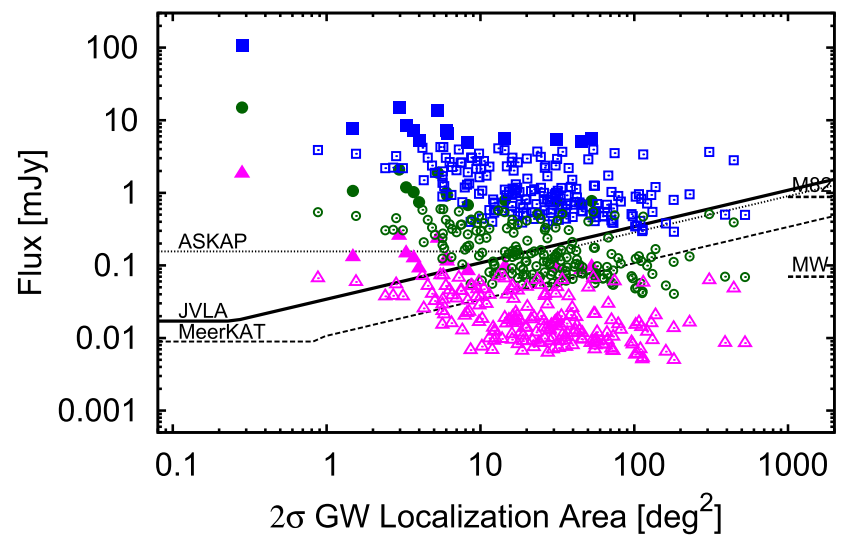

BH-NS, Net $5,1.4 \mathrm{GHz}, 30 \mathrm{hr}, 0.01 \mathrm{~cm}^{-3}$

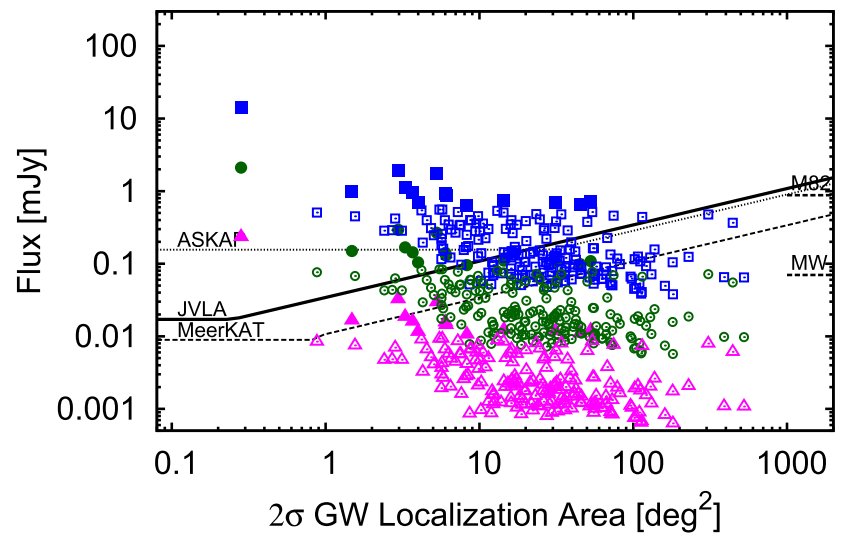

Figure 6. The same as Figure 3 but for the five-detector network.

et al. 1990) and these values are comparable to or even brighter than the expected luminosities of the radio counterparts (see Table 2). Here we discuss the probability that host galaxy contamination may prevent identification of GW-radio counterparts.

Galaxies bright in the radio band are either star-forming galaxies or those associated with AGNs. Since the former have radio emission extending spatially much more than the radio counterparts, i.e., small surface brightness, the contamination of a star-forming galaxy can be reduced significantly if the angular resolution of a radio facility is high enough to spatially resolve a galaxy. In contrast, AGNs and star-burst galaxies like M82 have compact radio emitting regions at their centers, i.e., large surface brightnesses. It is also important for mergers in these hosts to spatially resolve the galaxy scales to distinguish the radio counterparts from the compact core of the hosts. In the following, we discuss these different galaxy types of contamination separately.

For spatially extended sources like normal star-forming galaxies, the peak flux density $S_{p}$ in an image is the total flux 
ASKAP

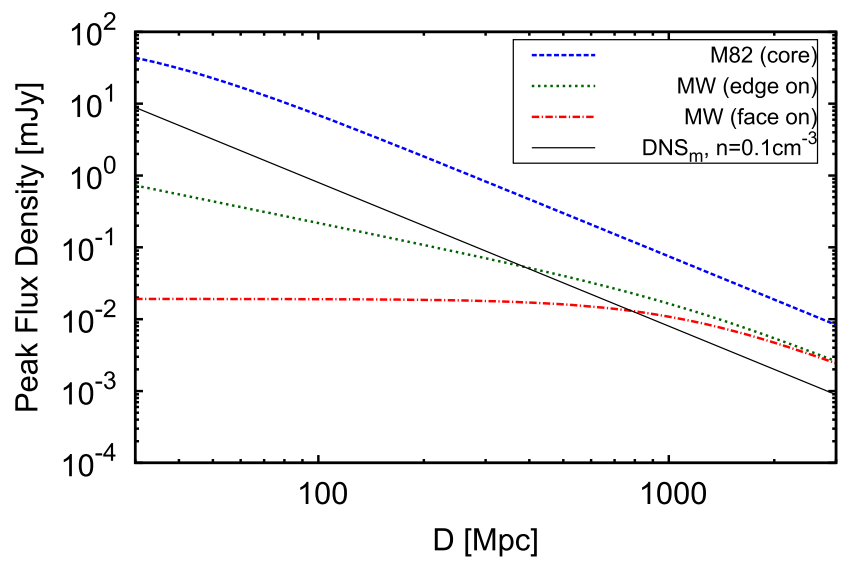

JVLA

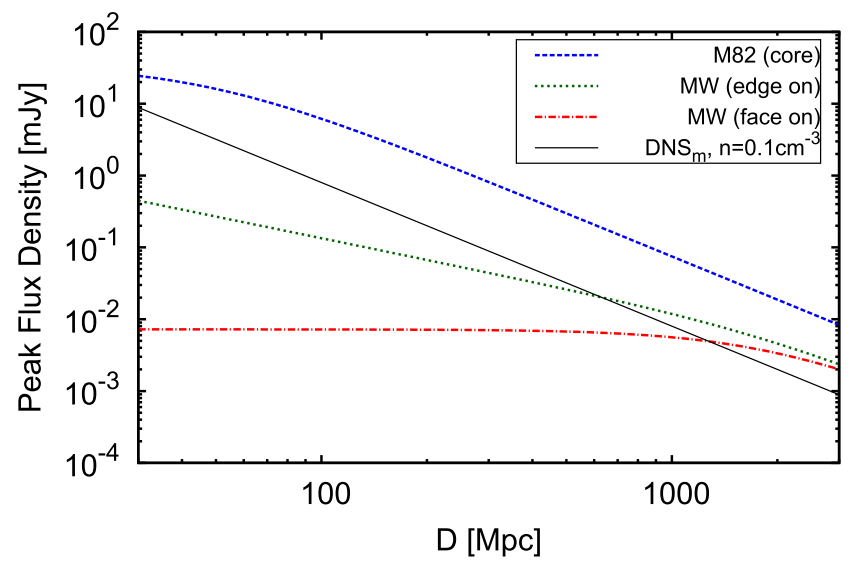

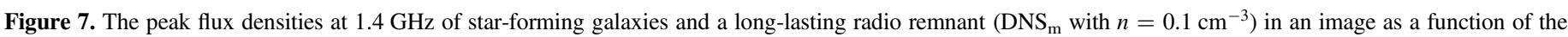

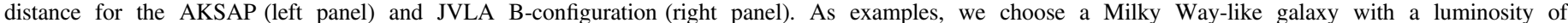

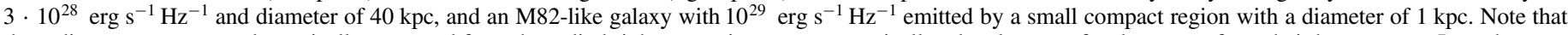

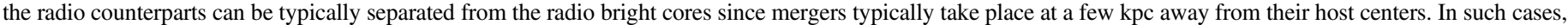
the counterparts are detectable. For a Milky Way-like galaxy, the face-on (dot-dashed) and edge-on (dotted) cases are shown.

density $S$ divided by the area (see e.g., Condon 2015). The peak flux density with angular resolution of $\theta$ is given by:

$$
\frac{S_{p}}{S}= \begin{cases}\left(\frac{\theta^{2}}{\theta^{2}+\phi^{2}}\right) & \text { (a face on galaxy), } \\ \left(\frac{\theta^{2}}{\theta^{2}+\phi^{2}}\right)^{1 / 2} & \text { (an edge on galaxy) }\end{cases}
$$

where $\phi$ is the angular diameter of a galaxy. A GW-radio counterpart is detectable if the ratio of its flux density to $S_{p}$ of the host is larger than a threshold which is determined by a false-positive probability based on the statistics of the variabilities of radio sources. Identifying radio counterparts will be possible when the flux densities of the counterparts are larger than the peak flux densities of the hosts.

In Figure 7, we show the peak flux density at $1.4 \mathrm{GHz}$ of a Milky Way-like galaxy with a luminosity of $3 \cdot 10^{28} \mathrm{erg} \mathrm{s}^{-1} \mathrm{~Hz}^{-1}$ and diameter of $40 \mathrm{kpc}$ (Beuermann et al. 1985), and an M82-like galaxy with a bright compact region with $10^{29} \mathrm{erg} \mathrm{s}^{-1} \mathrm{~Hz}^{-1}$ and $1 \mathrm{kpc}$ (Condon et al. 1990). Also shown is the peak flux density of a long-lasting radio remnant, $\mathrm{DNS}_{\mathrm{m}}$ with $n=0.1 \mathrm{~cm}^{-3}$, which is independent of the angular resolution of radio telescopes. For ASKAP, which has an angular resolution of 7", the peak flux density of $\mathrm{DNS}_{\mathrm{m}}$ with $n=0.1 \mathrm{~cm}^{-3}$ is brighter than those of Milky Way-like galaxies out to $800 \mathrm{Mpc}(300 \mathrm{Mpc})$ for the face-on (edge-on) case. For the JVLA B configuration $(\theta=4$ "! 3 ), the flux density of merger remnants are brighter than the peak flux densities of Milky Way-like galaxies out to a distance of $500 \mathrm{Mpc}$ even in the edge-on case. Thus, host contamination will not be a serious problem for Milky Way-like galaxies.

Star-burst galaxies and AGNs have bright radio emitting compact cores with a scale of $\sim 1 \mathrm{kpc}$. The radio counterparts in such hosts are identifiable if either they are spatially separated from bright compact cores or the counterparts themselves are brighter than these cores. It is important to note that more than $90 \%$ of sGRBs have projected physical offsets of $>1 \mathrm{kpc}$ from their host centers (Berger 2014). This suggests that mergers typically take place outside the core regions and will be detectable if a telescope has angular resolution high enough to separate a radio counterpart from the core of the host, e.g.,
$1 \mathrm{kpc}$ at $200 \mathrm{Mpc}$ corresponds to $\sim 1^{\prime \prime}$. For such a galaxy at $200 \mathrm{Mpc}$, the fractions of radio counterparts that are contaminated by the bright cores are estimated as $0.1,0.3$, and 0.7 for the JVLA A configuration $\left(\theta=11^{\prime \prime} 3\right)$, B configuration (4!" 3), and ASKAP $\left(7^{\prime \prime}\right)$ respectively. Here we use the distribution of projected physical offsets of sGRBs (Berger 2014).

Now we turn to estimate the population of galaxies that have peak flux densities brighter than a range of GW-radio counterparts. Based on the local radio luminosity function of star-forming galaxies (Condon et al. 2002), the number densities of galaxies brighter than $L_{1.4}\left(\mathrm{erg} \mathrm{s}^{-1} \mathrm{~Hz}^{-1}\right) \simeq\left(10^{27}, 10^{28}, 10^{29}\right)$ are estimated as $n_{>L}\left(\mathrm{Mpc}^{-3}\right) \simeq\left(7 \cdot 10^{-3}, 3 \cdot 10^{-3}, 3 \cdot 10^{-4}\right)$, respectively. Using the number density of galaxies of $n_{\mathrm{gal}} \simeq 0.01 \mathrm{Mpc}^{-3}$, the estimated fractions of star-forming galaxies brighter than $L_{1.4}=\left(10^{27}, 10^{28}, 10^{29}\right) \quad$ are $\quad f_{>_{L}} \simeq\left(\begin{array}{ll}0.7, & 0.3,0.03\end{array}\right)$, respectively. The same estimates can be done for AGNs using their radio luminosity function (Mauch \& Sadler 2007). The number densities of radio-bright AGNs are $n_{>L} \simeq\left(10^{-3}, 3 \cdot 10^{-4}, 10^{-4}\right)$ and the fractions are $f_{>L} \simeq(0.1,0.03,0.01)$. Therefore the majority of merger events likely take place in star-forming galaxies fainter than the Milky Way, for which host contamination will not be a serious problem; $5 \%-10 \%$ of events may occur in bright star-bursts and AGNs. Even for such cases, telescopes with high angular resolution can identify the radio counterparts by separating them from the radio-bright regions of hosts.

We can also estimate the population of radio-bright starforming galaxies hosting merger events based on the star formation rates (SFRs) of sGRB hosts. To do so, we use the phenomenological relation between the SFRs and the radio luminosities of star-forming galaxies: (Carilli \& Yun 1999; Condon et al. 2002):

$$
\begin{aligned}
L_{\nu} \approx & 1.2 \times 10^{28} \mathrm{erg} \mathrm{s}^{-1} \mathrm{~Hz}^{-1}\left(\frac{\mathrm{SFR}}{1 M_{\odot} \mathrm{yr}^{-1}}\right) \\
& \times\left(\frac{\nu}{1.4 \mathrm{GHz}}\right)^{-0.8},
\end{aligned}
$$


where a Kroupa initial mass function with $M \leqslant 100 M_{\odot}$ is assumed. Applying this relation to sGRB hosts of which their SFRs are estimated through the luminosities of the hosts in the rest-frame $B$-band (Berger et al. 2009; Berger 2014), the estimated fraction of galaxies hosting sGRBs brighter than $10^{28} \mathrm{erg} \mathrm{s}^{-1} \mathrm{~Hz}^{-1}$ is $\sim 0.5$. None of them is brighter than $10^{29} \mathrm{erg} \mathrm{s}^{-1} \mathrm{~Hz}^{-1}$ and fainter than $10^{27} \mathrm{erg} \mathrm{s}^{-1} \mathrm{~Hz}^{-1}$. These estimates are consistent with those estimated from the radio luminosity function of local galaxies.

We classify the radio counterparts as bright, marginal, and faint events as shown in the last column of Table 4 . Here we define the bright events as those with a luminosity of $L>10^{29} \mathrm{erg} \mathrm{s}^{-1} \mathrm{~Hz}^{-1}$, which is brighter than M82. The faint events are defined as those with a luminosity of $L<5 \cdot 10^{27} \mathrm{erg} \mathrm{s}^{-1} \mathrm{~Hz}^{-1}$, and the marginal events as those that have luminosities in between these values. The radio facilities with low angular resolution $\theta \sim 7^{\prime \prime}$ will be able to detect most of the medium events taking place in Milky Waylike galaxies.

\subsection{False Positives: Extragalactic Radio Transients and Variables}

Radio transients. There are various kinds of extragalactic astrophysical phenomena associated with relativistic or mildly relativistic explosions. They produce synchrotron radio emission on timescales of a week to years; see Metzger et al. (2015b) for a comprehensive study. Such events may incorrectly be identified as the radio counterparts of GW mergers. Radio transient surveys have already been conducted at flux densities up to $\sim 0.2 \mathrm{mJy}$ (e.g., Bannister et al. 2011; Ofek \& Frail 2011; Thyagarajan et al. 2011; Frail et al. 2012; Mooley et al. 2013, 2016). While many of them have not detected any radio transients, Bannister et al. (2011) have found 15 in $3000 \mathrm{deg}^{2}$ at $10 \mathrm{mJy}$. Mooley et al. (2016) also have found a few radio transients in $50 \mathrm{deg}^{2}$ at $0.2 \mathrm{mJy}$. Two of them are arising from Galactic flaring stars and the others likely from variable AGNs. The derived upper limit on the areal densities of extragalactic radio transients is $<0.4 \mathrm{deg}^{-2}$ for flux densities $\gtrsim 0.2 \mathrm{mJy}$ at $1.4 \mathrm{GHz}$ on timescales between a week and three months (Mooley et al. 2013) and $<0.04 \mathrm{deg}^{-2}$ for $\gtrsim 0.5 \mathrm{mJy}$ at $3 \mathrm{GHz}$ (Mooley et al. 2016). Therefore, we expect there to be less than one radio transient per square degree for flux densities of $\gtrsim 0.1 \mathrm{mJy}$. Thus, the number of radio false positive transients is much smaller than that of optical-infrared counterparts. For comparison, an areal density of extragalactic optical-infrared false positives at a depth of 24th apparent magnitude is $\sim 60 \mathrm{deg}^{-2}$ (e.g., NKG13).

We estimate the areal densities of radio transients based on the known radio bright astrophysical phenomena (see also Metzger et al. 2015b; Mooley et al. 2016). Table 5 summarizes relevant values of the radio transients used for the estimates of false positives. Figure 8 shows the expected areal densities of extragalactic radio transients brighter than $0.1 \mathrm{mJy}$ within given distances. The areal densities of type Ibc supernovae (SNe Ibc; Berger et al. 2003; Soderberg et al. 2006b), low-luminosity GRBs (LLGRB; Soderberg et al. 2006a; Barniol Duran et al. 2015), and tidal disruption events (TDEs) without strong jets (Alexander et al. 2015; Holoien et al. 2015; van Velzen et al. 2016) are so small that it will be quite rare to detect them as false positive transients. Although off-axis long GRBs (LGRBs; van Eerten et al. 2010; Ghirlanda et al. 2014)
Table 5

Astrophysical False Positive Transients

\begin{tabular}{lcccc}
\hline \hline Transients & $R\left(\mathrm{Gpc}^{-3} \mathrm{yr}^{-1}\right)$ & $\begin{array}{c}L_{1.4 \mathrm{GHz}} \\
\left(\mathrm{erg} \mathrm{s}^{-1} \mathrm{~Hz}^{-1}\right)\end{array}$ & $T$ (year) & Ref. \\
\hline Type II radio SN & $3 \cdot 10^{4}$ & $10^{27.5}$ & 10 & $(1)$ \\
SN Ib/c & 5000 & $10^{27}$ & 0.3 & $(2)$ \\
LLGRB & 500 & $5 \cdot 10^{27}$ & 0.1 & $(3)$ \\
Orphan LGRB & 15 & $2 \cdot 10^{29}$ & 3 & $(4)$ \\
TDE (strong jets) & 1 & $10^{31}$ & 3 & $(5)$ \\
TDE & 200 & $10^{28}$ & 0.5 & $(6)$ \\
\hline
\end{tabular}

References. (1) Levinson et al. (2002), Gal-Yam et al. (2006), Chevalier (1998), Weiler et al. (2002), (2) Berger et al. (2003), Soderberg et al. (2006b), (3) Soderberg et al. (2006a), Barniol Duran et al. (2015), (4) van Eerten et al. (2010), Ghirlanda et al. (2014), (5) Zauderer et al. (2011), Burrows et al. (2011), Berger et al. (2012), (6) van Velzen et al. (2016), Holoien et al. (2015), Alexander et al. (2015).

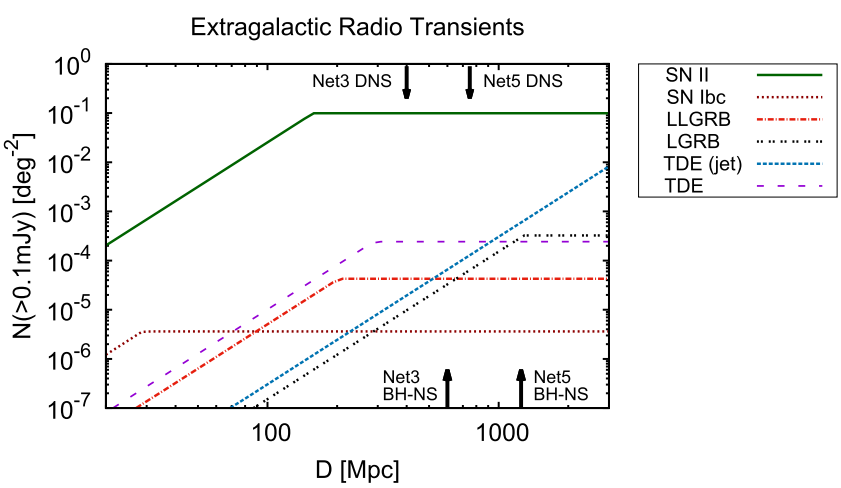

Figure 8. The areal densities of radio transients with flux densities brighter than $0.1 \mathrm{mJy}$ as a function of the source distance including: SNe II (solid; Chevalier 1998; Levinson et al. 2002; Weiler et al. 2002; Gal-Yam et al. 2006), SNe Ibc (dotted; Berger et al. 2003; Soderberg et al. 2006b), lowluminosity GRBs (dotted-dashed; Soderberg et al. 2006a; Barniol Duran et al. 2015), off-axis long GRBs (double-dotted; van Eerten et al. 2010; Ghirlanda et al. 2014), strong jet TDEs (dashed; Burrows et al. 2011; Zauderer et al. 2011; Berger et al. 2012; TDEs (long-dashed; Alexander et al. 2015; Holoien et al. 2015; van Velzen et al. 2016). Also shown are the maximum detectable distances of the GW networks for DNS and BH-NS mergers.

and tidal disruption events with strong jets (TDE (jet); Burrows et al. 2011; Zauderer et al. 2011; Berger et al. 2012) can be false positive transients, they will be identified earlier through their optical counterparts or can be filtered by identifying their host galaxies since the typical distance of these events is far beyond the detectable distance of the GW networks.

A certain fraction of type II supernovae have bright radio luminosities of $10^{26}-10^{28} \mathrm{erg} \mathrm{s}^{-1} \mathrm{~Hz}^{-1}$ on timescales of 100-1000 days (Chevalier 1998; Weiler et al. 2002). According to the identification of a radio supernova in a radio survey without any other counterparts (Levinson et al. 2002; Gal-Yam et al. 2006), the areal density of radio supernovae is roughly estimated as $0.1 \mathrm{deg}^{-2}$ at $0.1 \mathrm{mJy}$. Thus there will be a few to tens of type II radio supernovae in a GW localization field. There are several ways to identify radio supernovae. First, they can be clearly identifiable as supernovae if the associated supernovae are observable in the optical bands. The ongoing and upcoming optical transient surveys are powerful methods to ensure prior optical identification of such supernovae. A fraction of supernovae, however, will be missed in optical surveys due to strong dust extinction. Indeed, a supernova SN 2008iz in M82 is discovered only in the radio bands 

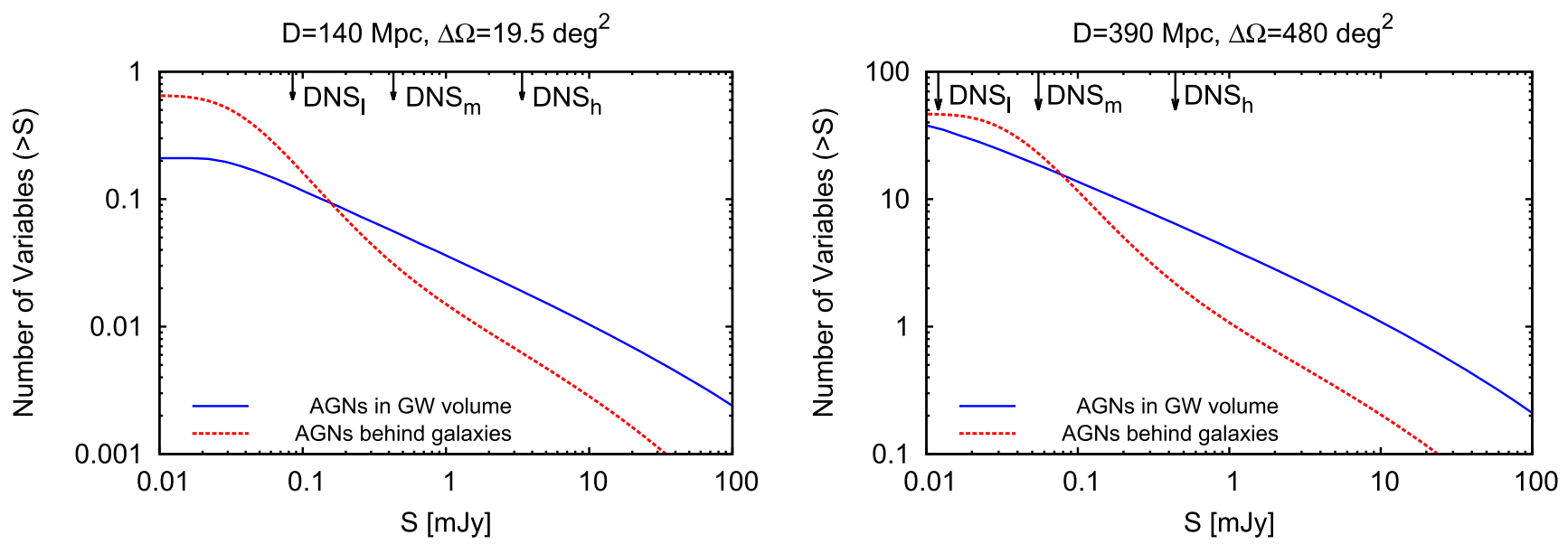

Figure 9. The number of radio variables behind the galaxies within a GW localized volume at $1.4 \mathrm{GHz}$ as a function of the flux densities. Two specific cases for DNS mergers using GW Net 3 are shown: a merger at a distance of $140 \mathrm{Mpc}$ with a localization area of $19.5 \mathrm{deg}^{2}$ (an optimistic case; left panel) and that at $390 \mathrm{Mpc}$ with $480 \mathrm{deg}^{2}$ (a pessimistic case; right panel). Also shown are the flux densities of $\mathrm{DNS}_{\mathrm{h}}, \mathrm{DNS}_{\mathrm{m}}$, and $\mathrm{DNS}_{1}$ with a circum-binary density of $0.1 \mathrm{~cm}^{-3}$. Note that this analysis does not incorporate the degree of variability. In reality, fewer AGNs contribute to false positives for brighter radio counterparts (see the text for details).

(Brunthaler et al. 2009, 2010). Even if associated supernovae are not identifiable, they can be distinguished from merger events using timescale arguments, which are significantly longer than those of GW-radio counterparts, and radio spectral properties. Because radio bright supernovae take place in high circumstellar densities, their radio spectra are affected strongly by synchrotron self-absorption and free-free absorption. Such strong absorption features should be absent in the radio signals arising from compact binary mergers at frequencies above $1 \mathrm{GHz}$.

Variable radio sources. The observed flux densities of persistent extragalactic radio sources vary with time due to either intrinsic variabilities or interstellar scintillation. If the variability of these sources are large enough $(\gtrsim 30 \%)$ on timescales between days and a few years, they will be detected as false positives. According to radio variable studies (e.g., Ofek et al. 2011; Thyagarajan et al. 2011; Mooley et al. 2013, 2016), the population of radio variables on these timescales with flux densities at $1.4 \mathrm{GHz}$ between $0.3 \mathrm{mJy}$ and $100 \mathrm{mJy}$ is about $1 \%$ or less of the total persistent radio sources and these variables are mainly AGNs. The areal density of persistent radio point sources with flux densities larger than $0.1 \mathrm{mJy}$ is $\sim 1000 \mathrm{deg}^{-2}$ (Huynh et al. 2005) and roughly half of them are AGNs, therefore hundreds to thousands of radio variables are expected to be in GW localization areas. Most variable AGNs can be rejected by using their redshift information that will be beyond their GW localization volumes. However, some fraction of them will remain as false positives and they are divided into two groups: (i) AGNs inside the GW localization volumes and (ii) AGNs outside the GW localization volumes but behind the host galaxy candidates. In what follows, we discuss these two cases of AGN false positives separately.

As discussed in Section 5.1, the number of AGNs inside the GW localization volumes can be estimated based on the local radio luminosity function of AGNs (Mauch \& Sadler 2007), which gives $\sim 3 \mathrm{deg}^{-2}(D / 450 \mathrm{Mpc})^{3}$ at $0.1 \mathrm{mJy}$. Given a fraction of variable sources $\lesssim 1 \%$, the number of false positives due to radio variables inside the $\mathrm{GW}$ localization volumes is $\lesssim 3(D / 450 \mathrm{Mpc})^{3}\left(\Delta \Omega_{\mathrm{GW}} / 100 \mathrm{deg}^{2}\right)$ at $0.1 \mathrm{mJy}$. Figure 9 shows the number of these false positives as a function of the flux densities (blue solid lines) for two examples using GW Net
3: a well-localized merger event (an optimistic case) at $D=140 \mathrm{Mpc}$ with $\Delta \Omega_{\mathrm{GW}}=19.5 \mathrm{deg}^{2}$ (left panel) and a poorly localized one (a pessimistic case) at $D=390 \mathrm{Mpc}$ and with $\Delta \Omega_{\mathrm{GW}}=480 \mathrm{deg}^{2}$ (right panel). Here we assume $1 \%$ of AGNs are variable. Also shown are the flux densities of $\mathrm{DNS}_{\mathrm{h}}$, $\mathrm{DNS}_{\mathrm{m}}$, and $\mathrm{DNS}_{1}$ with a circum-binary density of $0.1 \mathrm{~cm}^{-3}$. At the flux densities of these models, the expected number of the false positives is $6-40$ for the poorly localizable GW events. In contrast, this number is significantly reduced as $0.02-0.1$ for the well-localizable GW event because of a relatively small GW localization volume: $\Delta \Omega_{\mathrm{GW}}=19.5 \mathrm{deg}^{2} \quad$ and $D=139_{-21}^{+79} \mathrm{Mpc}$.

AGNs outside the GW localization volumes but behind the host galaxy candidates of the GW merger events will prove more problematic. Assuming each galaxy has a disk shape with a diameter of $50 \mathrm{kpc}, \sim 1 \%$ of the sky is covered by galaxies inside a distance of $450 \mathrm{Mpc}$ so that we expect the number of the false positives due to AGNs behind those galaxies to be $\sim 5\left(\Delta \Omega_{\mathrm{GW}} / 100 \mathrm{deg}^{2}\right)$ at $0.1 \mathrm{mJy}$. The expected number of radio variables behind galaxies inside of the GW localization volumes as a function of the flux densities is shown in Figure 9 (red lines). Here the population of the background radio sources derived by Huynh et al. (2005) is used. The sky areas covered by the host galaxy candidates within the GW localization volumes are estimated using the number density of galaxies $0.01 \mathrm{Mpc}^{-3}$. The number of the false positives linearly declines with the flux densities around $0.1 \mathrm{mJy}$. For the poorly localizable case, the expected numbers of these variables are 1, 20 , and 50 at the flux densities of $\mathrm{DNS}_{\mathrm{h}}, \mathrm{DNS}_{\mathrm{m}}$, and $\mathrm{DNS}_{1}$ with $n=0.1 \mathrm{~cm}^{-3}$ respectively. For the well-localizable case, these values are $6 \cdot 10^{-3}, 0.03$, and 0.2 .

Some of the false positives due to AGNs are removable using multi-epoch observations if they do not fade away. In addition, there are several ways to identify AGNs using: (i) radio source catalogs which will be available thanks to existing and upcoming radio all-sky surveys, (ii) the locations of the radio counterparts in the host galaxies compared to the AGN central cores, and (iii) AGNs have flat radio spectra around $1 \mathrm{GHz}$, which are different from those of the radio counterparts. Here method (ii) is valid only for AGNs inside the GW localization volumes. Note that the analysis here does not incorporate the degree of variability. The population of variable 
AGNs decreases with the modulation index $\Delta S / S=2\left|S_{1}-S_{2}\right| /\left(S_{1}+S_{2}\right)$, where $S_{1}$ and $S_{2}$ are the flux densities in two epochs. For instance, Mooley et al. (2016) find that only one out of 3700 radio sources is highly variable as $\Delta S / S \approx 1$, thereby the number of false positives due to radio variables is significantly reduced for brighter radio counterparts. Note that, however, the population of radio variable sources depends on the sensitivity, observed frequency, timescale, and direction of the sky and such analysis still remains unqualified at the flux densities of GW-radio counterparts. Therefore a critical understanding of the properties of radio variable sources is necessary to identify $\mathrm{GW}$ radio counterparts, especially in the era of GW astronomy where we may have tens of GW detections per year.

The above discussions are based on the assumption that we have a galaxy catalog covering the $\mathrm{GW}$ localization volumes. This significantly reduces the number of false positives. However, the spectroscopic galaxy catalogs currently available are incomplete, in particular beyond $200 \mathrm{Mpc}$. This incompleteness, which is not so critical for DNS merges, will be crucial for identifying the radio counterparts to $\mathrm{BH}-\mathrm{NS}$ mergers, of which the detectable distances in GWs are as high as $1 \mathrm{Gpc}$. Therefore, deeper optical observations will be necessary to complete galaxy catalogs out to the edge of the GW localization volumes when identifying the radio-GW counterparts for such cases.

\section{COMPARISON WITH PREVIOUS WORKS}

To compare our radio counterpart detectability results with previous works we translate the detection likelihood to a detection rate for a given merger rate density $R$. For $\mathrm{DNS}_{\mathrm{m}}$ with $n=0.1 \mathrm{~cm}^{-3}$, the expected radio detection rates are $\sim 7$ and $20 \mathrm{yr}^{-1}\left(R / 500 \mathrm{Gpc}^{-3} \mathrm{yr}^{-1}\right)$ for the JVLA and MeerKAT in GW Net 3 (see Equation (7) in NKG13). Metzger et al. (2015b) studied the detectability of extragalactic radio transients and found the expected detection rate of long-lasting radio remnants arising from the DNS merger ejecta to be $\lesssim 0.03 \mathrm{yr}^{-1}\left(R / 500 \mathrm{Gpc}^{-3} \mathrm{yr}^{-1}\right)$ for a three-year survey with ASKAP and JVLA. This rate is much lower than the one we find for a number of reasons. Our work focuses on the followup surveys of GW merger events so that the observations are optimized and can reach a sensitivity as deep as $\sim 0.1 \mathrm{mJy}$. In contrast, Metzger et al. (2015b) considered blind surveys that can detect radio transients with much higher flux densities of 1-5 mJy. Moreover, many events are missed by Metzger et al. (2015b) due to the variability criterion for detections because the peak timescale of the signals is too long compared to the duration of the surveys, i.e., one cannot recognize the radio signals as transients. This reduces the detection rate by an order of magnitude. Note also that the ejecta model of Metzger et al. (2015b) is an outflow with a single velocity component with $v=0.2 c$, which gives a longer peak timescale and a fainter peak flux density than an outflow with multi-velocity components as given by Equation (7). If one takes the ejectas' multi-velocity components into account, the detectability of neutron star binary mergers in blind surveys may increase.

Long-lived magnetars have been proposed to explain the prompt GRB emission or late-time X-ray activities of sGRBs based either on the spin-down luminosity (Fan \& Xu 2006; Troja 2007; Metzger et al. 2008; Rowlinson et al. 2013; Gao et al. 2015; Lü et al. 2015; Siegel \& Ciolfi 2016a, 2016b), or on outflows powered by differential rotation (Shibata et al. 2011;
Kiuchi et al. 2012; Siegel et al. 2014). In fact, numerical simulations have recently shown strong amplification of magnetic fields at merger (Price \& Rosswog 2006; Giacomazzo \& Perna 2013; Giacomazzo et al. 2015; Kiuchi et al. 2015). The spin-down magnetar model predicts that the ejecta can derive a large amount of kinetic energy $\sim 10^{52} \mathrm{erg}$ from the magnetar itself. For typical merger ejecta masses, such ejecta expand with relativistic velocities and result in bright radio emissions.

Metzger \& Bower (2014) constrained such magnetar models using late-time radio observations of sGRBs and ruled out a magnetar remnant in GRB 050724 and 060505. More recently, Horesh et al. (2016) provided strong constraints on magnetar activity of the macronova candidates: GRB 060614 and 130603B. In addition, radio transient surveys can put strong constraints on the the formation rate of the magnetars (Metzger et al. 2015b). The current limit on the rate is about $5 \mathrm{Gpc}^{-3} \mathrm{yr}^{-1}$, which is already much lower than the expected NS merger rate by one to two orders of magnitude. At the typical distance of GW merger events, the expected radio flux densities of the magnetar models are $\sim 100-1000 \mathrm{mJy}$ so that the radio follow-up observations will easily detect such signals. Moreover, these signals are sufficiently brighter than the typical radio luminosity of the galaxies and false positive transients and variables. Therefore, identification of post-merger magnetar emission from GW events will be relatively straightforward.

\section{RECOMMENDATIONS FOR THE RADIO SURVEYS}

Here we briefly summarize our recommendations for the radio surveys. We propose that radio follow-up observations of $\mathrm{GW}$ mergers at $1.4 \mathrm{GHz}$ take place in five epochs separated by logarithmic time intervals: within a day after the detection, at $\lesssim 10$ days, at $\sim 30$ days, at $\sim 100$ days, at $\sim 300$ days and at $\gtrsim 1000$ days. At $3 \mathrm{GHz}$, a similar strategy can be employed. The radio surveys at $3 \mathrm{GHz}$ have the advantage of high angular resolution, which reduces the radio contamination of the host galaxies, while the peak fluxes are lower than those at $1.4 \mathrm{GHz}$ by a factor of $\sim 2$. At $150 \mathrm{MHz}$, we recommend conducting intensive surveys at late times (a year or later) because the radio light curves at the low frequencies arise at later times. Importantly, the peak flux densities are higher than those at 1.4 GHz. Therefore, the late-time observations at $150 \mathrm{MHz}$ will be quite important for both the detections and confirmations of the GW radio counterparts. Finally, a comparison with the optical data will be necessary to identify the redshift of the radio counterpart candidates, otherwise astrophysical false positives, mainly radio variable AGNs, contaminate significantly.

\section{SUMMARY AND DISCUSSION}

We have explored optimized strategies for detecting longlasting radio signals arising from compact binary mergers following the detection of $\mathrm{GW}$ events. To do so, we first simulated GW merger events and constructed mock catalogs of detectable GW events. We then computed the expected radio flux densities assuming different ejecta models and circummerger densities for each detectable $\mathrm{GW}$ event. We focused on synchrotron radiation arising from (i) sub-relativistic merger ejecta (long-lasting radio remnants) and (ii) ultra-relativistic jets (orphan GRB afterglows). The radio flux densities depend on the unknown ejectas' kinetic energy and velocity 
distributions and the circum-merger densities. Here we take into account uncertainties in the ejectas' kinetic energy and velocities according to numerical simulations of compact binary mergers and three different circum-merger densities of $0.01,0.1$, and $1 \mathrm{~cm}^{-3}$ based on the Galactic DNS population.

Based on the derived light curves, we propose that radio follow-up observations of GW mergers at $1.4 \mathrm{GHz}$ take place in five epochs separated by logarithmic time intervals: within a day after the detection, at $\lesssim 10$ days, at $\sim 30$ days, at $\sim 100$ days, at $\sim 300$ days and at $\gtrsim 1000$ days. We compare the expected radio flux density of each GW merger event with the sensitivities of a slew of radio facilities assuming that each radio telescope searches the radio counterparts in the $\mathrm{GW}$ localization areas. Assuming a total observation time of $30 \mathrm{hr}$ for each epoch, we derive the detection likelihood of each ejecta model. Note that we have not taken into account Northern and Southern hemisphere considerations of the GW sky localizations, and hence the subsequent relative detectability fractions should be reduced by approximately a factor of two. For the sub-relativistic merger ejecta, the JVLA will detect $5 \%-90 \%$ of the GW events with a GW network of three detectors for circum-merger densities of $0.1 \mathrm{~cm}^{-3}$. For the orphan GRB afterglows with a canonical (large) kinetic energy, $\sim 10$ (40) $\%$ of the GW events will be detected by the JVLA. We find that the detection likelihood does not change significantly for a $\mathrm{GW}$ network with five detectors because the gain in the radio sensitivity due to better localizations somehow compensates for the loss in radio flux densities due to the increase in the GW detectable distance.

The detection likelihood increases if we conduct follow-up observations only for the well-localizable GW events. For instance, the JVLA can detect more than $60 \%$ (15\%) of the GW events for which localization areas are better than $20 \mathrm{deg}^{2}$ for the ejecta model with medium kinetic energies, velocities, and a density of $0.1 \mathrm{~cm}^{-3}\left(0.01 \mathrm{~cm}^{-3}\right)$. Note that the probability of localizing a GW DNS merger to within a sky area of $20 \mathrm{deg}^{2}$ is about $25 \%$ for a GW network of three detectors.

The detectable radio signals at $150 \mathrm{MHz}$ appear at later times (around three years or later) and the sensitivity of widefield searches may be limited by confusion noise. However, it is quite important to search for the radio counterparts at low frequency since their spectral properties are quite different from those of other radio transients and variables, e.g., radio supernovae and AGNs. Such observations will be significant to discriminate between radio counterparts from other astrophysical false positives. Therefore, we suggest that lowfrequency arrays, such as LOFAR, search with high angular resolution for the radio counterparts of $\mathrm{GW}$ events for which the radio counterpart candidates have been detected earlier by other radio facilities at higher frequencies.

Looking into the next decade, the Square Kilometer Array (SKA) will achieve much faster survey speeds than those used in this work. It will hence detect radio counterparts of GW mergers far more efficiently. Here we discuss prospects for SKA-mid and focus on the detectability prospects based on $\mathrm{DNS}_{\mathrm{m}}$ at $1.4 \mathrm{GHz}$ with the GW Net 5. In order to detect most of the radio counterparts of $\mathrm{GW}$ merger events at $n=0.1 \mathrm{~cm}^{-3}$, the required survey speed is 100 times faster than that of the JVLA. The survey speeds of SKA-mid will reach this value. Therefore we expect a significant progress in studies of radio counterparts once SKA and GW Net 5 both become operational.
We discuss the possible contamination of the host galaxies, which can be divided into normal star-forming galaxies that spatially extend on a radial scale of $\sim 10 \mathrm{kpc}$ and AGNs and star-bursts that have central radio bright compact regions on a radial scale of $\sim 1 \mathrm{kpc}$. For both cases, resolving the hosts will greatly reduce the contamination. For instance, the radio counterparts are identifiable for $\mathrm{DNS}_{\mathrm{m}}$ with a density of $0.1 \mathrm{~cm}^{-3}$ in Milky Way-like galaxies out to $400 \mathrm{Mpc}$. While the probability is low, mergers may take place in radio-bright AGNs and star-bursts. In order to be identifiable, the radio counterparts must be separable spatially from the galaxies' bright compact regions. For such a galaxy at $200 \mathrm{Mpc}$, we estimate that the fractions that the radio counterpart are masked due to galaxy contamination are $0.1,0.3$, and 0.7 for the JVLA A configuration, B configuration, and ASKAP respectively.

Astrophysical false positives, including extragalactic radio transients and variables, may mimic the radio counterparts. We estimate that a few to tens of radio transients will be detectable at $0.1 \mathrm{mJy}$ as false positives and most of them may be type II supernovae. These false positives can be rejected using the optical identification of supernovae, their longer timescales, and strong-absorption features in the radio spectra.

The false positives due to radio variables will be more problematic. A few hundreds to thousands of variables (mainly AGNs) will be detectable at flux densities comparable to the detectable GW-radio counterparts in a GW localization area of $\sim 100 \mathrm{deg}^{2}$. Most of these false-positives will be located at distances far beyond the GW detectable distance and they can be rejected as merger candidates by identifying their distances or redshifts. However, there will be a few to tens of radio variables in the GW-localization volumes. Moreover, some fraction of radio variables beyond the GW-horizon distance will be located behind the host galaxy candidates. They can be rejected using the multi-epoch variability test, the location of the host, and the flatter spectra than those of the radio counterparts. It is worth emphasizing that the number of false positives is significantly reduced for well-localized events. We expect there to be less than one false positive for such an event. Note the number of false positives due to variables per unit sky area depends on the sensitivity, degree of variability, observed frequency, and the sky direction, so that a better understanding of the statistical properties of radio variables will be important for identifying radio counterparts.

In summary, while there are uncertainties in the ejectas' kinetic energy, velocity, and circum-merger density, a certain fraction of GW merger events will be detectable with current and upcoming radio facilities. In addition, identifying radio counterparts will not be so difficult thanks to the relatively quite radio transient sky. We therefore advocate radio counterpart surveys for GW merger events for which EM counterparts in other wavelengths (e.g., X-ray and optical) have not been detected, using not only the GW sky error but the GW distance and galaxy catalogs, thanks to the relatively long timescales of the radio emission.

This work was supported by the I-CORE Program of the Planning and Budgeting Committee and The Israel Science Foundation (grant No 1829/12) and by an ISF-CNSF grant. SMN acknowledges generous support from the Radboud University Excellence Fellowship. Part of this research was carried out at the Jet Propulsion Laboratory, California Institute 


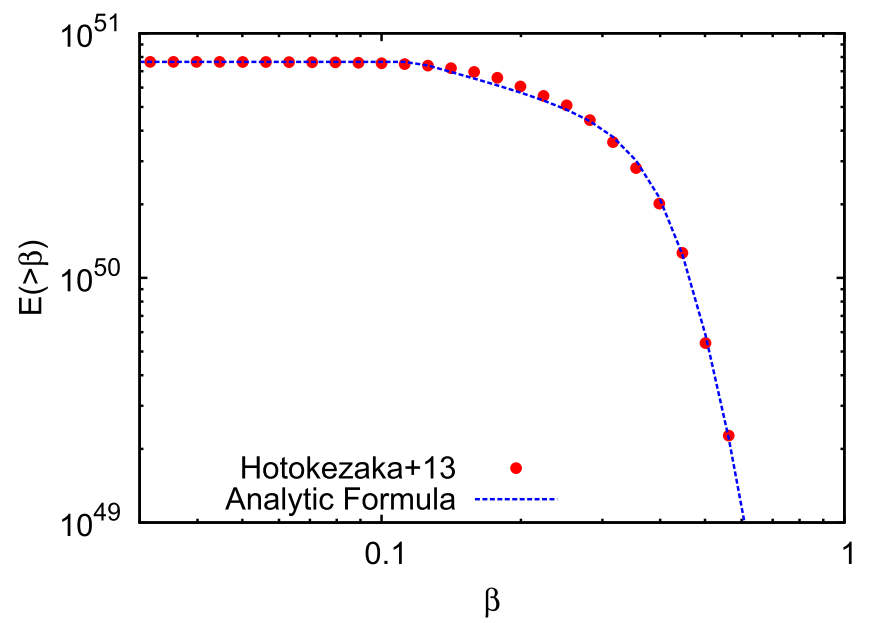

Figure 10. Ejecta kinetic energy above a given velocity. Red points show a result of a numerical relativity simulation by Hotokezaka et al. (2013). Also shown as a blue line is an analytic formula given by Equation (7).

of Technology, under a contract with the National Aeronautics and Space Administration.

We are very grateful to Dale Frail for a careful reading of the manuscript and for initially encouraging us to embark on this project. We thank Keith Bannister, Paz Beniamin, Robert Braun, Jess Broderick, Aaron Chippendale, Assaf Horesh, Rob Fender, Jason Hessels, David Kaplan, Mansi Kasliwal, Shri Kulkarni, Duncan Lorimer, Kunal Mooley, Tara Murphy, Steve Myers, and Antonia Rowlinson for useful discussions. We thank the anonymous referee for suggestions that improved our work.

\section{APPENDIX}

We show the ejecta kinetic energy profile in Figure 10 taken from a numerical-relativity simulation by Hotokezaka et al. (2013). Here a result of an equal-mass DNS merger simulation with a total mass of $2.8 M_{\odot}$ and a neutron star equation of state APR4 is shown as an example. We also depict an analytic formula, a power law with an exponential cut-off (see Equation (7)). It can be seen that this formula describes the result of the simulation well. Note that a significant fraction of material, more than $25 \%$ in terms of the kinetic energy, has velocities above $0.4 c$. These fast components contribute to the radio light curves at early times. As a result, the flux densities at early times are brighter than those expected from an ejecta model with a single velocity component.

\section{REFERENCES}

Abadie, J., Abbott, B. P., Abbott, R., et al. 2010, CQGra, 27, 173001 Abbott, B. P., Abbott, R., Abbott, T. D., et al. 2016a, arXiv:1602.08492 Abbott, B. P., Abbott, R., Abbott, T. D., et al. 2016b, PhRvL, 116, 061102 Abbott, B. P., Abbott, R., Adhikari, R., et al. 2009, RPPh, 72, 076901 Acernese, F., Agathos, M., Agatsuma, K., et al. 2015, CQGra, 32, 024001 Alexander, K. D., Berger, E., Guillochon, J., Zauderer, B. A., \& Williams, P. K. G. 2016, ApJL, 819, L25

Bannister, K. W., Murphy, T., Gaensler, B. M., Hunstead, R. W., \& Chatterjee, S. 2011, MNRAS, 412, 634

Barnes, J., \& Kasen, D. 2013, ApJ, 775, 18

Barniol Duran, R., Nakar, E., Piran, T., \& Sari, R. 2015, MNRAS, 448, 417 Bauswein, A., Ardevol Pulpillo, R., Janka, H.-T., \& Goriely, S. 2014, ApJL, 795, L9
Bauswein, A., Goriely, S., \& Janka, H.-T. 2013, ApJ, 773, 78

Belczynski, K., Perna, R., Bulik, T., et al. 2006, ApJ, 648, 1110

Beniamini, P., \& Piran, T. 2016, MNRAS, 456, 4089

Berger, E. 2014, ARA\&A, 52, 43

Berger, E., Cenko, S. B., Fox, D. B., \& Cucchiara, A. 2009, ApJ, 704, 877

Berger, E., Fong, W., \& Chornock, R. 2013, ApJL, 774, L23

Berger, E., Kulkarni, S. R., Frail, D. A., \& Soderberg, A. M. 2003, ApJ, 599, 408

Berger, E., Zauderer, A., Pooley, G. G., et al. 2012, ApJ, 748, 36

Berry, C. P. L., Mandel, I., Middleton, H., et al. 2015, ApJ, 804, 114

Beuermann, K., Kanbach, G., \& Berkhuijsen, E. M. 1985, A\&A, 153, 17

Blanchet, L. 2014, LRR, 17, 2

Booth, R. S., \& Jonas, J. L. 2012, AfrSk, 16, 101

Brunthaler, A., Martí-Vidal, I., Menten, K. M., et al. 2010, A\&A, 516, A27

Brunthaler, A., Menten, K. M., Reid, M. J., et al. 2009, A\&A, 499, L17

Burrows, D. N., Kennea, J. A., Ghisellini, G., et al. 2011, Natur, 476, 421

Cannon, K., Cariou, R., Chapman, A., et al. 2012, ApJ, 748, 136

Carilli, C. L., \& Yun, M. S. 1999, ApJL, 513, L13

Chevalier, R. A. 1998, ApJ, 499, 810

Chu, Q., Howell, E. J., Rowlinson, A., et al. 2016, MNRAS, 459, 121

Condon, J. 2015, arXiv:1502.05616

Condon, J. J., Cotton, W. D., \& Broderick, J. J. 2002, AJ, 124, 675

Condon, J. J., Helou, G., Sanders, D. B., \& Soifer, B. T. 1990, ApJS, 73, 359

Coward, D. M., Howell, E. J., Piran, T., et al. 2012, MNRAS, 425, 2668

Cutler, C., \& Flanagan, É. E. 1994, PhRvD, 49, 2658

De Colle, F., Ramirez-Ruiz, E., Granot, J., \& Lopez-Camara, D. 2012, ApJ, 751, 57

Dominik, M., Berti, E., O’Shaughnessy, R., et al. 2015, ApJ, 806, 263

Draine, B. T. 2011, Physics of the Interstellar and Intergalactic Medium (Princeton, NJ: Princeton Univ. Press)

Eichler, D., Livio, M., Piran, T., \& Schramm, D. N. 1989, Natur, 340, 126

Fairhurst, S. 2010, CQG, 28, 105021

Fan, Y.-Z., \& Xu, D. 2006, MNRAS, 372, L19

Feng, L., Vaulin, R., \& Hewitt, J. N. 2014, arXiv:1405.6219

Finn, L. S. 1992, PhRvD, 46, 5236

Fong, W., Berger, E., Margutti, R., \& Zauderer, B. A. 2015, ApJ, 815, 102

Fong, W., Berger, E., Metzger, B. D., et al. 2014, ApJ, 780, 118

Foucart, F. 2012, PhRvD, 86, 124007

Foucart, F., Deaton, M. B., Duez, M. D., et al. 2013, PhRvD, 87, 084006

Frail, D. A., Kulkarni, S. R., Ofek, E. O., Bower, G. C., \& Nakar, E. 2012, ApJ, 747, 70

Frail, D. A., Soderberg, A. M., Kulkarni, S. R., et al. 2005, ApJ, 619, 994

Frail, D. A., Waxman, E., \& Kulkarni, S. R. 2000, ApJ, 537, 191

Gal-Yam, A., Ofek, E. O., Poznanski, D., et al. 2006, ApJ, 639, 331

Gao, H., Zhang, B., \& Lü, H.-J. 2015, PhRvD, 93, 044065

Gehrels, N., Cannizzo, J. K., Kanner, J., et al. 2016, ApJ, 820, 136

Ghirlanda, G., Burlon, D., Ghisellini, G., et al. 2014, PASA, 31, 22

Giacomazzo, B., \& Perna, R. 2013, ApJL, 771, L26

Giacomazzo, B., Zrake, J., Duffell, P. C., MacFadyen, A. I., \& Perna, R. 2015, ApJ, 809, 39

Granot, J., Panaitescu, A., Kumar, P., \& Woosley, S. E. 2002, ApJL, 570, L61

Granot, J., \& Piran, T. 2012, MNRAS, 421, 570

Granot, J., Piran, T., \& Sari, R. 1999, ApJ, 527, 236

Grover, K., Fairhurst, S., Farr, B. F., et al. 2014, PhRvD, 89, 042004

Guetta, D., \& Piran, T. 2005, A\&A, 435, 421

Guetta, D., \& Piran, T. 2006, A\&A, 453, 823

Hansen, B. M. S., \& Lyutikov, M. 2001, MNRAS, 322, 695

Heald, G. H., Pizzo, R. F., Orrú, E., et al. 2015, A\&A, 582, A123

Holoien, T. W.-S., Kochanek, C. S., Prieto, J. L., et al. 2015, MNRAS, 455, 2918

Horesh, A., Hotokezaka, K., Piran, T., Nakar, E., \& Hancock, P. 2016a, ApJL, 819, L22

Hotokezaka, K., Kiuchi, K., Kyutoku, K., et al. 2013, PhRvD, 87, 024001

Hotokezaka, K., \& Piran, T. 2015, MNRAS, 450, 1430

Hotokezaka, K., Piran, T., \& Paul, M. 2015, NatPh, 11, 1042

Flanagan, É. É., \& Hughes, S. A. 1998, PhRvD, 57, 4535

Huynh, M. T., Jackson, C. A., Norris, R. P., \& Prandoni, I. 2005, AJ, 130,1373

Intema, H. T., Jagannathan, P., Mooley, K. P., \& Frail, D. A. 2016, arXiv: 1603.04368

Jin, Z.-P., Hotokezaka, K., Li, X., et al. 2016, arXiv:1603.07869

Johnston, S., Taylor, R., Bailes, M., et al. 2008, ExA, 22, 151

Just, O., Bauswein, A., Pulpillo, R. A., Goriely, S., \& Janka, H.-T. 2015, MNRAS, 448, 541

Kasliwal, M. M. 2011, PhD thesis, California Institute of Technology

Kasliwal, M. M., \& Nissanke, S. 2014, ApJL, 789, L5 
Kawaguchi, K., Kyutoku, K., Nakano, H., et al. 2015, PhRvD, 92, 024014 Kiel, P. D., Hurley, J. R., \& Bailes, M. 2010, MNRAS, 406, 656

Kiuchi, K., Cerdá-Durán, P., Kyutoku, K., Sekiguchi, Y., \& Shibata, M. 2015, PhRvD, 92, 124034

Kiuchi, K., Kyutoku, K., \& Shibata, M. 2012, PhRvD, 86, 064008

Klimenko, S., Vedovato, G., Drago, M., et al. 2011, PhRvD, 83, 102001

Kochanek, C. S., \& Piran, T. 1993, ApJL, 417, L17

Komatsu, E., Dunkley, J., Nolta, M. R., et al. 2009, ApJS, 180, 330

Kulkarni, S. R. 2005, arXiv:astro-ph/0510256

Kyutoku, K., Ioka, K., Okawa, H., Shibata, M., \& Taniguchi, K. 2015, PhRvD, 92, 044028

Kyutoku, K., Ioka, K., \& Shibata, M. 2014, MNRAS, 437, L6

Levinson, A., Ofek, E. O., Waxman, E., \& Gal-Yam, A. 2002, ApJ, 576, 923

Lewis, A., \& Bridle, S. 2002, PhRvD, 66, 103511

Li, L.-X., \& Paczyński, B. 1998, ApJL, 507, L59

Li, M., Ostriker, J. P., Cen, R., Bryan, G. L., \& Naab, T. 2015, ApJ, 814, 4 Lorimer, D. R. 2008, LRR, 11, 8

Lü, H.-J., Zhang, B., Lei, W.-H., Li, Y., \& Lasky, P. D. 2015, ApJ, 805, 89

Margalit, B., \& Piran, T. 2015, MNRAS, 452, 3419

Mauch, T., \& Sadler, E. M. 2007, MNRAS, 375, 931

Metzger, B. D., Bauswein, A., Goriely, S., \& Kasen, D. 2015a, MNRAS, 446, 1115

Metzger, B. D., \& Berger, E. 2012, ApJ, 746, 48

Metzger, B. D., \& Bower, G. C. 2014, MNRAS, 437, 1821

Metzger, B. D., Martínez-Pinedo, G., Darbha, S., et al. 2010, MNRAS, 406, 2650

Metzger, B. D., Quataert, E., \& Thompson, T. A. 2008, MNRAS, 385, 1455

Metzger, B. D., Williams, P. K. G., \& Berger, E. 2015b, ApJ, 806, 224

Mooley, K. P., Frail, D. A., Ofek, E. O., et al. 2013, ApJ, 768, 165

Mooley, K. P., Hallinan, G., Bourke, S., et al. 2016, ApJ, 818, 105

Nakar, E. 2007, PhR, 442, 166

Nakar, E., Gal-Yam, A., \& Fox, D. B. 2006, ApJ, 650, 281

Nakar, E., \& Piran, T. 2011, Natur, 478, 82

Nakar, E., Piran, T., \& Granot, J. 2002, ApJ, 579, 699

Narayan, R., Paczynski, B., \& Piran, T. 1992, ApJL, 395, L83

Nissanke, S., Holz, D. E., Hughes, S. A., Dalal, N., \& Sievers, J. L. 2010, ApJ, 725,496

Nissanke, S., Kasliwal, M., \& Georgieva, A. 2013, ApJ, 767, 124

Nissanke, S., Sievers, J., Dalal, N., \& Holz, D. 2011, ApJ, 739, 99

Ofek, E. O., \& Frail, D. A. 2011, ApJ, 737, 45

Ofek, E. O., Frail, D. A., Breslauer, B., et al. 2011, ApJ, 740, 65

Oosterloo, T., Verheijen, M., \& van Cappellen, W. 2010, in ISKAF2010 Science Meeting (Assen: Proc. of Science), 43

Paczynski, B. 1991, AcA, 41, 257

Palenzuela, C., Lehner, L., Ponce, M., et al. 2013, PhRvL, 111, 061105

Perley, R. A., Chandler, C. J., Butler, B. J., \& Wrobel, J. M. 2011, ApJL, 739, L1

Piran, T., Korobkin, O., \& Rosswog, S. 2014, arXiv:1401.2166

Piran, T., Nakar, E., \& Rosswog, S. 2013, MNRAS, 430, 2121

Price, D. J., \& Rosswog, S. 2006, Sci, 312, 719

Pshirkov, M. S., \& Postnov, K. A. 2010, Ap\&SS, 330, 13

Radice, D., Galeazzi, F., Lippuner, J., et al. 2016, MNRAS, 460, 3255
Roberts, L. F., Kasen, D., Lee, W. H., \& Ramirez-Ruiz, E. 2011, ApJL, 736, L21

Rodriguez, C. L., Farr, B., Raymond, V., et al. 2014, ApJ, 784, 119

Rosswog, S. 2005, ApJ, 634, 1202

Rosswog, S. 2013, RSPTA, 371, 20272

Rowlinson, A., O'Brien, P. T., Metzger, B. D., Tanvir, N. R., \& Levan, A. J. 2013, MNRAS, 430, 1061

Sari, R., Piran, T., \& Narayan, R. 1998, ApJL, 497, L17

Schutz, B. F. 2011, CQGra, 28, 125023

Sekiguchi, Y., Kiuchi, K., Kyutoku, K., \& Shibata, M. 2015, PhRvD, 91, 064059

Sekiguchi, Y., Kiuchi, K., Kyutoku, K., Shibata, M., \& Taniguchi, K. 2016, PhRvD, 93, 124046

Shen, S., Cooke, R. J., Ramirez-Ruiz, E., et al. 2015, ApJ, 807, 115

Shibata, M., Suwa, Y., Kiuchi, K., \& Ioka, K. 2011, ApJL, 734, L36

Shimwell, T. W., Luckin, J., Brüggen, M., et al. 2016, MNRAS, 459, 277

Siegel, D. M., \& Ciolfi, R. 2016a, ApJ, 819, 14

Siegel, D. M., \& Ciolfi, R. 2016b, ApJ, 819, 15

Siegel, D. M., Ciolfi, R., \& Rezzolla, L. 2014, ApJL, 785, L6

Singer, L. P., Chen, H.-Y., Holz, D. E., et al. 2016, arXiv:1603.07333

Singer, L. P., Price, L. R., Farr, B., et al. 2014, ApJ, 795, 105

Soderberg, A. M., Kulkarni, S. R., Nakar, E., et al. 2006a, Natur, 442, 1014

Soderberg, A. M., Nakar, E., Berger, E., \& Kulkarni, S. R. 2006b, ApJ, 638, 930

Tanaka, M., \& Hotokezaka, K. 2013, ApJ, 775, 113

Tanvir, N. R., Levan, A. J., Fruchter, A. S., et al. 2013, Natur, 500, 547

The LIGO Scientific Collaboration \& the Virgo Collaboration 2016, arXiv: 1602.03840

The LIGO Scientific Collaboration, the Virgo Collaboration, Abbott, B. P., et al. 2013, arXiv: 1304.0670

Thyagarajan, N., Helfand, D. J., White, R. L., \& Becker, R. H. 2011, ApJ, 742, 49

Tingay, S. J., Goeke, R., Bowman, J. D., et al. 2013, PASA, 30, e007

Totani, T. 2013, PASJ, 65, L12

Troja, E. 2007, ApJ, 665, 599

van de Voort, F., Quataert, E., Hopkins, P. F., Kereš, D., \& Faucher-Giguère, C.-A. 2015, MNRAS, 447, 140

van Eerten, H., Zhang, W., \& MacFadyen, A. 2010, ApJ, 722, 235

van Haarlem, M. P., Wise, M. W., Gunst, A. W., et al. 2013, A\&A, 556, A2

van Velzen, S., Anderson, G. E., Stone, N. C., et al. 2016, Sci, 351, 62

Vangioni, E., Goriely, S., Daigne, F., François, P., \& Belczynski, K. 2016, MNRAS, 455,17

Veitch, J., Mandel, I., Aylott, B., et al. 2012, PhRvD, 85, 104045

Veitch, J., Raymond, V., Farr, B., et al. 2015, PhRvD, 91, 042003

Voss, R., \& Tauris, T. M. 2003, MNRAS, 342, 1169

Wanderman, D., \& Piran, T. 2015, MNRAS, 448, 3026

Wehmeyer, B., Pignatari, M., \& Thielemann, F.-K. 2015, MNRAS, 452, 1970

Weiler, K. W., Panagia, N., Montes, M. J., \& Sramek, R. A. 2002, ARA\&A, 40, 387

Weisberg, J. M., Nice, D. J., \& Taylor, J. H. 2010, ApJ, 722, 1030

Wen, L., \& Chen, Y. 2010, PhRvD, 81, 082001

Yang, B., Jin, Z.-P., Li, X., et al. 2015, NatCo, 6, 7323

Zauderer, B. A., Berger, E., Soderberg, A. M., et al. 2011, Natur, 476, 425 